\title{
\begin{tabular}{l|l} 
Mitraries & DSpace@MIT
\end{tabular}
}

\author{
MIT Open Access Articles
}

Extracting Design Principles for Efficient Thermally Activated Delayed Fluorescence (TADF) from a Simple Four-State Model

The MIT Faculty has made this article openly available. Please share how this access benefits you. Your story matters.

Citation: Silva, Piotr de et al. “Extracting Design Principles for Efficient Thermally Activated Delayed Fluorescence (TADF) from a Simple Four-State Model." Chemistry of materials 31 (2019): 6995-7006.

As Published: https://dx.doi.org/10.1021/ACS.CHEMMATER.9B01601

Publisher: American Chemical Society (ACS)

Persistent URL: https://hdl.handle.net/1721.1/125454

Version: Original manuscript: author's manuscript prior to formal peer review

Terms of Use: Article is made available in accordance with the publisher's policy and may be subject to US copyright law. Please refer to the publisher's site for terms of use. 


\section{Extracting Design Principles for Efficient Thermally Activated Delayed Fluorescence (TADF) from a Simple Four-State Model}

Piotr de Silva, Changhae Andrew Kim, Tianyu Zhu, Troy Van Voorhis

Submitted date: 16/03/2019 - Posted date: 18/03/2019

Licence: CC BY-NC-ND 4.0

Citation information: de Silva, Piotr; Kim, Changhae Andrew; Zhu, Tianyu; Van Voorhis, Troy (2019):

Extracting Design Principles for Efficient Thermally Activated Delayed Fluorescence (TADF) from a Simple Four-State Model. ChemRxiv. Preprint.

We introduce a simple quantum-mechanical model for thermally activated delayed fluorescence (TADF). The Hamiltonian is represented in the basis of four spin-mixed diabatic states representing pure charge transfer (CT) and local excitations (LE). The model predicts that it is possible to realize lowest-lying adiabatic singlet (S1) and triplet (T1) states with a small singlet triplet gap, differing CT/LE contributions, and appreciable LE component in the $\mathrm{S} 1$ state. These characteristics can explain the coexistence of fast $\mathrm{T} 1 \rightarrow \mathrm{S} 1$ reverse intersystem crossing and $\mathrm{S} 1 \rightarrow \mathrm{S} 0$ radiative decay in some chromophores. Through the sampling of the parameter space and statistical analysis of the data, we show which parameter combinations contribute the most to the TADF efficiency. We also show that conformational fluctuations of a single model donor-acceptor system sample a significant region of the parameter space and can enhance the TADF rate by almost three orders of magnitude. This study provides new guidelines for optimization of TADF emitters by means of electronic structure and conformation engineering.

File list (2)

Extracting Design Principles for Efficient Thermally Activat... (0.98 MiB) view on ChemRxiv • download file SI Extracting Design Principles for Efficient Thermally Activ... (1.03 MiB) view on ChemRxiv • download file 


\title{
Extracting Design Principles for Efficient Thermally Activated Delayed Fluorescence (TADF) from a Simple Four-State Model
}

\author{
Piotr de Silva ${ }^{* \ddagger}$, Changhae Andrew Kim ${ }^{\dagger}$, Tianyu Zhu ${ }^{\dagger}$, Troy Van Voorhis ${ }^{* \dagger}$ \\ ${ }^{\dagger}$ Department of Chemistry, 77 Massachusetts Avenue, Massachusetts Institute of Technology, \\ Cambridge, MA 02139, USA. \\ ${ }^{\ddagger}$ Department of Energy Conversion and Storage, Technical University of Denmark, \\ Fysikvej 309, 2800 Kgs. Lyngby, Denmark \\ *e-mail: pdes@dtu.dk \\ *e-mail: tvan@mit.edu
}

\begin{abstract}
We introduce a simple quantum-mechanical model for thermally activated delayed fluorescence (TADF). The Hamiltonian is represented in the basis of four spin-mixed diabatic states representing pure charge transfer (CT) and local excitations (LE). The model predicts that it is possible to realize lowest-lying adiabatic singlet $\left(\mathrm{S}_{1}\right)$ and triplet $\left(\mathrm{T}_{1}\right)$ states with a small singlet-triplet gap, differing CT/LE contributions, and appreciable LE component in the $S_{1}$ state. These characteristics can explain the coexistence of fast $\mathrm{T}_{1} \rightarrow \mathrm{S}_{1}$ reverse intersystem crossing and $\mathrm{S}_{1} \rightarrow \mathrm{S}_{0}$ radiative decay in some chromophores. Through the sampling of the parameter space and statistical analysis of the data, we show which parameter combinations contribute the most to the TADF efficiency. We also show that conformational fluctuations of a single model donor-acceptor system sample a significant region of the parameter space and can enhance the TADF rate by almost three orders of magnitude. This study provides new guidelines for optimization of TADF emitters by means of electronic structure and conformation engineering.
\end{abstract}



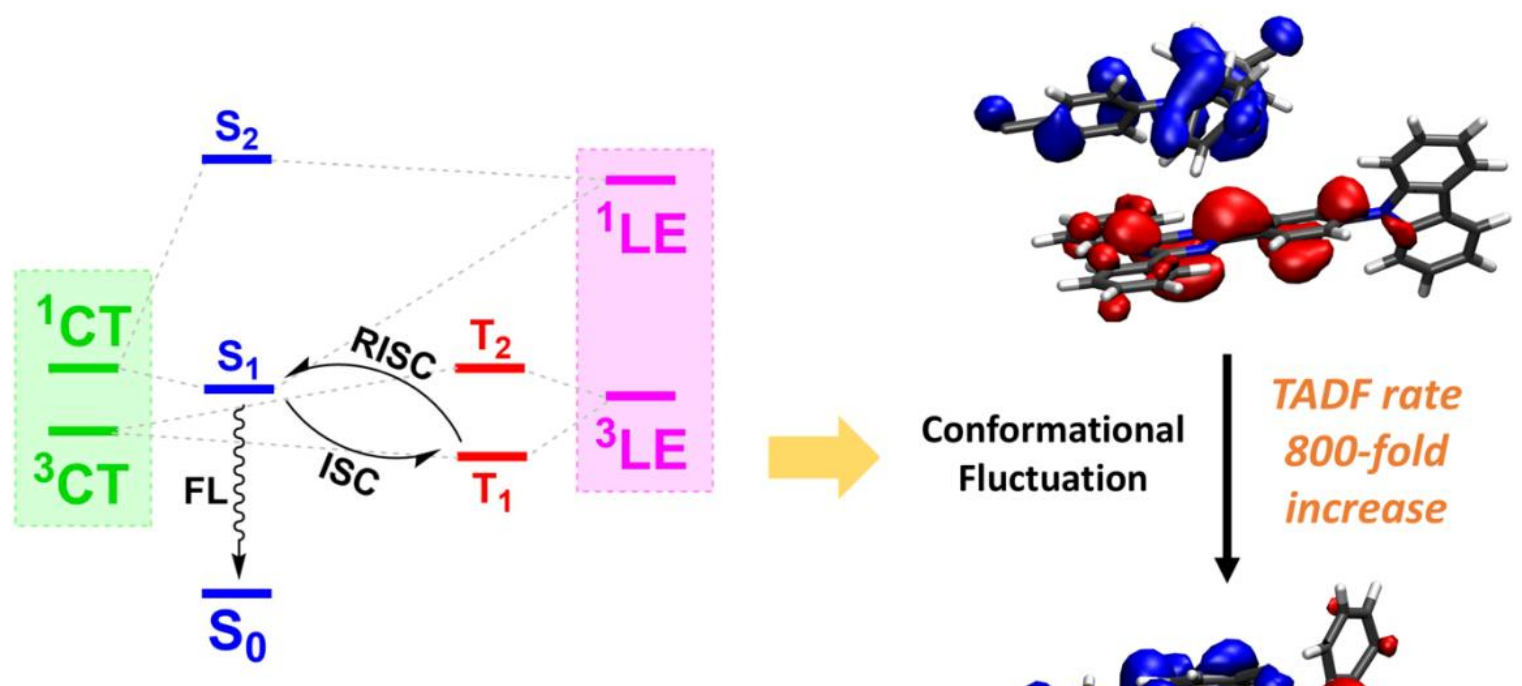

TADF Four-State Model

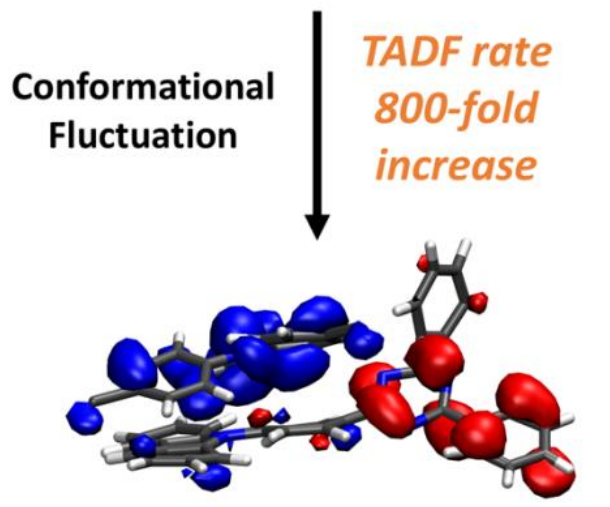




\section{Introduction}

Thermally activated delayed fluorescence (TADF) ${ }^{1,2}$ is believed to be one of the most promising routes to increase the efficiency of organic light-emitting diode (OLED) devices. ${ }^{3-8}$ Harvesting of normally nonemissive triplet excitons is achieved through design of molecular emitters that can undergo an efficient thermally activated intersystem crossing to a singlet manifold, followed by a radiative relaxation to the ground state. To date, this idea has enabled a large number of metal-free organic emitter ${ }^{9-19}$ and the technology is becoming competitive with already commercially deployed phosphorescent OLEDs. ${ }^{11,16,18-21}$ In an OLED device, $25 \%$ of generated excitons end up in the first excited singlet state $S_{1}$ and $75 \%$ in the first excited triplet state $T_{1}$. Due to electron exchange interactions, the dark $T_{1}$ state has lower energy than the bright $S_{1}$ state (Figure 1a). In the canonical model of TADF, the triplet exciton needs to be thermally activated to become isoenergetic with the $\mathrm{S}_{1}$ state. Subsequently, spin-orbit $(\mathrm{SO})$ coupling can convert it into a singlet state, from which photon emission is a spin-allowed process. This reverse intersystem crossing (RISC) mechanism is a thermally activated process, so its efficiency critically depends on the energy barrier, i.e. the singlet-triplet gap $\Delta E_{S T}$. Additionally, the rate of transition depends on the spin-

orbit coupling $\left\langle S_{1}\left|\widehat{H}_{S O}\right| T_{1}\right\rangle$ between the relevant states. This highlights that efficient RISC can proceed, assuming small but non-vanishing spin-orbit coupling, only if $S_{1}$ and $T_{1}$ are energetically close.

The first successful purely organic TADF emitters were based on the design principle aiming at minimization of the singlet-triplet gap ${ }^{5-7}$. This was achieved by linking electron donor and acceptor units in such a way that the dihedral angle was close to orthogonality. As a result, the highest occupied molecular orbital (HOMO) and lowest unoccupied molecular orbital (LUMO) were localized on the donor and acceptor, respectively. The minimization of the HOMO-LUMO spatial overlap led to small exchange splitting, and consequently small $\Delta E_{S T}$. Employing this donor-acceptor (D-A) architecture resulted in a 
strong charge transfer (CT) character of the lowest excited singlet and triplet states, best described by a one-electron HOMO to LUMO excitation.

To date, there have been many ab initio studies of TADF emitters, initially focusing on calculations of the singlet-triplet gaps ${ }^{22-28}$. High-throughput screening approaches have been proposed ${ }^{29-31}$ to find new emitters with an effective tradeoff between small $\Delta E_{S T}$ and fast radiative decay. Further experimental ${ }^{15,32-34}$ studies have shown that for many TADF molecules the lowest triplet state is in fact a local excitation (LE), which indicated that the understanding of the TADF mechanism is incomplete and known design strategies are not optimal. Since then, a number of theoretical works have analyzed the mixed CT/LE nature of the excited states ${ }^{35-40}$, importance of vibronic effects ${ }^{41-45}$, and influence of the interactions with the environment ${ }^{46-49}$, including the importance of static and dynamic disorder ${ }^{50,51}$. Several recent review articles summarize the computational and theoretical developments in the field ${ }^{52-}$ 55.

In this paper, we propose a new four-state model for TADF that generalizes the canonical model by including both the CT and LE states. We examine the behavior of this model as a function of its microscopic parameters, i.e. the energy gap and electronic couplings between the states. In the Theory section, we begin by summarizing the canonical two-state model and then introduce the four-state generalization. Next, in the Results section, we perform a Monte Carlo sampling of the parameter space to unravel which parameters are decisive for TADF efficiency. This is followed by an ab initio exploration of the conformation space of a model donor-acceptor system. The study allows us to formulate new guidelines for the design of efficient TADF materials.

\section{Theory}

Two-state model of TADF 
The standard two-state model for $\operatorname{TADF}^{7}$ can be described as an electron transfer from the HOMO orbital localized on the donor moiety to the LUMO orbital on the acceptor. This picture gives rise to two isoenergetic spin-mixed charge-transfer states CT1 and CT2

$$
\begin{aligned}
& |C T 1\rangle=\frac{1}{\sqrt{2}}\left|\phi_{H}(1) \alpha(1) \phi_{L}(2) \beta(2)\right| \\
& |C T 2\rangle=-\frac{1}{\sqrt{2}}\left|\phi_{H}(1) \beta(1) \phi_{L}(2) \alpha(2)\right|
\end{aligned}
$$

The electronic coupling between these two states is known as the exchange integral

$$
\langle C T 1|\widehat{H}| C T 2\rangle=\left(\phi_{H} \phi_{L} \mid \phi_{L} \phi_{H}\right)=K_{H L}
$$

and the corresponding eigenfunctions of the Hamiltonian are spin-pure states $\left|{ }^{1} C T\right\rangle=\frac{1}{\sqrt{2}}(|C T 1\rangle+$ $|C T 2\rangle)$ and $\left|{ }^{3} C T\right\rangle=\frac{1}{\sqrt{2}}(|C T 1\rangle-|C T 2\rangle)$. The energies of these states can be written as

$$
\begin{aligned}
& E_{S_{1}}=h_{H}+h_{L}+J_{H L}+K_{H L} \\
& E_{T_{1}}=h_{H}+h_{L}+J_{H L}-K_{H L}
\end{aligned}
$$

where $h_{H} / h_{L}$ is the one-electron HOMO/LUMO integrals and $J_{H L}$ is the Coulomb two-electron integral $\left(\phi_{H} \phi_{H} \mid \phi_{L} \phi_{L}\right)$. From Eqs. 4 and 5 results that the singlet-triplet gap is simply twice the exchange integral

$$
\Delta E_{S T}=2 K_{H L}
$$

which immediately suggest that minimizing the exchange integral by spatial separation of HOMO and LUMO is a reasonable design strategy for TADF emitters.

This design principle has been realized in many TADF emitters which are based on the donor-acceptor (DA) architecture ${ }^{11,18}$. The large dihedral angle between D and A units causes spatial localization of HOMO and LUMO and consequently small exchange integral $K_{H L}$. Despite these early successes, it has been realized that the two-state model has several deficiencies. First, a pure ${ }^{1} C T$ state would have a very small oscillator strength, so efficient luminescence following efficient RISC would not be possible. This is 
because there is a single variable (HOMO-LUMO overlap) that governs both the oscillator strength and singlet-triplet gap. Efficient TADF requires both large oscillator strength (large HOMO-LUMO overlap) and a small singlet triplet gap (small HOMO-LUMO overlap). There is thus an inescapable tradeoff - one cannot have high oscillator strength and small gaps at the same time - and so TADF rates are fundamentally limited to a theoretical maximum of $\sim 1 \mu \mathrm{s}^{-1}$. The second deficiency of the two-state model is that ISC between two states of the same electronic character is very inefficient as the spin flip needs to be offset by a change of the orbital angular momentum so that the total angular momentum is conserved ${ }^{56,57}$. This means that the spin-orbit coupling between two pure CT states vanishes $\left\langle{ }^{1} \mathrm{CT}\left|\mathrm{H}_{S O}\right|{ }^{3} \mathrm{CT}\right\rangle=0$ and the intersystem crossing is impossible irrespective of how small the energy gap is. Finally, it has been noticed that TADF efficiency does not always correlate with the small singlet-triplet gap ${ }^{10}$. Relatively high efficiencies have been observed for emitters with large apparent gaps. Also, donor-acceptor-donor (D-AD) architectures have been shown to be more efficient compared to D-A systems despite having similar singlet-triplet gaps ${ }^{8,9,58}$. Recently, several new approaches to the design of TADF emitters ${ }^{16,59,60}$ and increasing the efficiency of the devices ${ }^{20,61,62}$ have been proposed.

\section{Four-state model of TADF}

In the two-state model, efficient TADF is limited because of the tradeoff between small singlet-triplet gaps and large oscillator strengths due to the $\mathrm{CT}$ character of the states involved. On the other hand, there is experimental evidence that a local triplet $\left({ }^{3} \mathrm{LE}\right)$ state that is energetically close to both CT states plays a role in $\mathrm{RISC}^{15,32-34}$. In particular, it has been suggested that vibronic non-adiabatic effects are responsible for the interaction between CT and LE states and efficient RISC ${ }^{41-44,57,63-65}$. Here we propose a model that generalizes the two-state model to include also LE states. This model bypasses the need for non-adiabatic effects to explain efficient TADF; however, it does not preclude this mechanism to be operational in some cases. 
As the starting point, we assume that the active space is composed of three orbitals. To focus the discussion, we assume that $\mathrm{HOMO}$ and LUMO+1 are localized on the donor, and LUMO is localized on the acceptor ( 2 electrons in 3 orbitals); however, the model works for any three orbitals that can describe one-electron charge-transfer and local excitations (e.g. HOMO on donor and HOMO-1, LUMO on acceptor -4 electrons in 3 orbitals). Consideration of all possible single excitations within the active space gives four electron configurations illustrated in the top panel of Figure $1 \mathrm{~b}$. These configurations correspond to the conceptual picture of excitations as the electron transfer between spatially localized molecular orbitals.
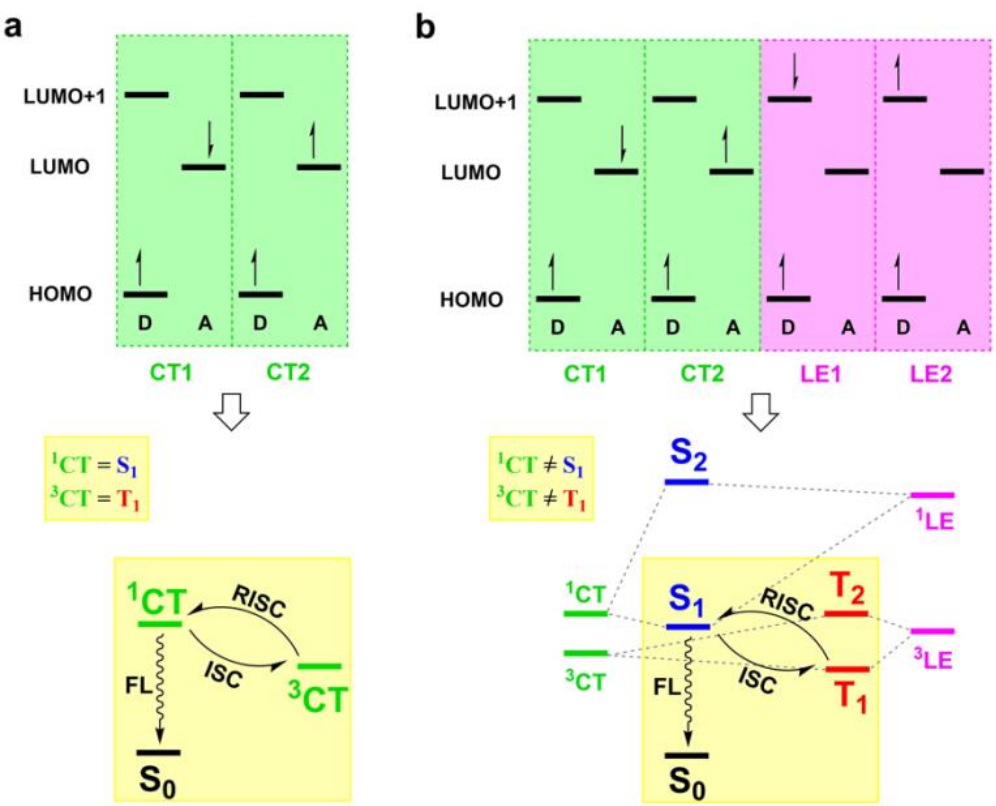

Figure 1 Comparison of electronic configurations and resulting energy diagrams in a two-state (a) and four-state (b) model.

Note that these states are diabatic states with a well-defined electronic character, so they can be classified as charge-transfer (CT1 and CT2) or local (LE1 and LE2) excitations, and that they are not eigenfunctions of the spin operator. For these reasons, the Hamiltonian in the $(|C T 1\rangle,|C T 2\rangle,|L E 1\rangle,|L E 2\rangle)$ basis is not diagonal and, to a good approximation (discussed in the SI), takes the following form 


$$
\widehat{H}=\left(\begin{array}{cccc}
0 & K_{C T} & t & K_{X} \\
K_{C T} & 0 & K_{X} & t \\
t & K_{X} & \Delta E & K_{L E} \\
K_{X} & t & K_{L E} & \Delta E
\end{array}\right)
$$

The parameters of the Hamiltonian are $\Delta E$ - the energy gap between CT and LE states, where the energy of the CT states is arbitrarily set to 0 ; the exchange integrals

$$
\begin{gathered}
K_{C T}=\langle C T 1|\widehat{H}| C T 2\rangle=\left(\phi_{H} \phi_{L} \mid \phi_{L} \phi_{H}\right) \\
K_{L E}=\langle L E 1|\widehat{H}| L E 2\rangle=\left(\phi_{H} \phi_{L+1} \mid \phi_{L+1} \phi_{H}\right)
\end{gathered}
$$

a sum of a one-electron hopping integral and a two-electron integral

$$
t=\langle C T 1|\widehat{H}| L E 1\rangle=t^{\prime}+K_{X}^{\prime}=\left\langle\phi_{L}|\hat{h}| \phi_{L+1}\right\rangle+\left(\phi_{H} \phi_{H} \mid \phi_{L} \phi_{L+1}\right)
$$

where $\hat{h}$ is the one-electron part of the Hamiltonian; and a two-electron integral

$$
K_{X}=\langle C T 1|\widehat{H}| L E 2\rangle=\left(\phi_{H} \phi_{L+1} \mid \phi_{L} \phi_{H}\right)
$$

The Hamiltonian in Eq. 7 can be block-diagonalized in the basis of diabatic spin-pure states

$$
\begin{aligned}
\left.\left.\right|^{1} C T\right\rangle & =\frac{1}{\sqrt{2}}(|C T 1\rangle+|C T 2\rangle) \\
\left.\left.\right|^{3} C T\right\rangle & =\frac{1}{\sqrt{2}}(|C T 1\rangle-|C T 2\rangle) \\
\left.\left.\right|^{1} L E\right\rangle & =\frac{1}{\sqrt{2}}(|L E 1\rangle+|L E 2\rangle) \\
\left.\left.\right|^{3} L E\right\rangle & =\frac{1}{\sqrt{2}}(|L E 1\rangle-|L E 2\rangle)
\end{aligned}
$$

which leads to two $2 \times 2$ Hamiltonians operating in the singlet and triplet subspaces

$$
\begin{aligned}
& \widehat{H}_{S}=\left(\begin{array}{cc}
K_{C T} & t+K_{X} \\
t+K_{X} & \Delta E+K_{L E}
\end{array}\right) \\
& \widehat{H}_{T}=\left(\begin{array}{cc}
-K_{C T} & t-K_{X} \\
t-K_{X} & \Delta E-K_{L E}
\end{array}\right)
\end{aligned}
$$


Diagonalizing these Hamiltonians gives closed form expressions for the energies of the adiabatic states $E_{S 1}, E_{S 2}, E_{T 1}, E_{T 2}$, as well as for the coefficients of the eigenvectors in the basis of spin-pure diabatic states (see SI). In particular, the expression for the singlet-triplet energy gap $\Delta E_{S T}$ is the following

$$
\begin{gathered}
\Delta E_{S T}=K_{L E}+K_{C T}-\sqrt{\frac{1}{4}\left(\Delta E+K_{L E}+K_{C T}\right)^{2}-\left(\Delta E+K_{L E}\right) K_{C T}+\left(t+K_{X}\right)^{2}} \\
+\sqrt{\frac{1}{4}\left(\Delta E-K_{L E}-K_{C T}\right)^{2}+\left(\Delta E-K_{L E}\right) K_{C T}+\left(t-K_{X}\right)^{2}}
\end{gathered}
$$

which is significantly more complicated than simply twice the exchange integral within the two-state model. The gap $\Delta E_{S T}$ depends on all the parameters of the model and it is clear that the design principle based on the minimization of HOMO-LUMO spatial overlap is not the only way to obtain a small singlettriplet gap. The first square root in Eq. 18 reduces the gap, while the second acts in the opposite direction, so the final outcome depends on the interplay between $\Delta E$ and different coupling elements. While the exchange couplings and $\Delta E$ affect the energy separation between CT and LE diabatic states, the mixing of these diabats is determined by $\left|t+K_{X}\right|$ and $\left|t-K_{X}\right|$ for singlet and triplet manifolds, respectively. This means that CT and LE states of different multiplicity have different mixing strengths, which will result in a different electronic character of the respective energy states. Due to the different contribution of CT and LE states, the spin-orbit coupling between $S_{1}$ and $T_{1}$ states could be appreciable without the mediation of the $T_{2}$ state via non-adiabatic coupling. The efficiency of this direct pathway will depend on the relative signs and values of $t$ and $K_{X}$, as well as values of $\Delta E$ and exchange integrals. Coupling of ${ }^{1} C T$ and ${ }^{1} L E$ through the $\left(t+K_{X}\right)$ element also means that the $S_{1}$ state is a mixture of the CT and LE diabats rather than a pure CT state, which explains how TADF molecules can have an appreciable dipole coupling to the ground state and be efficient light emitters (see Figure 1b).

At this point, the four-state model explains why efficient TADF is possible in organic molecules: In principle, the right electronic Hamiltonian can simultaneously yield a small singlet-triplet gap $\Delta E_{S T}$ together with 
qualitatively different contributions of LE and CT states in the $S_{1}$ and $T_{1}$ adiabats, which enable efficient inter-system crossing, as well as a significant contribution of the local excitation in the $\mathrm{S}_{1}$ state, which enables high quantum yields. This clearly demonstrates that the tradeoff between minimizing the HOMOLUMO overlap for small singlet-triplet gaps and maximizing it for efficient fluorescence is not a physical limitation, but merely an artifact of the oversimplified two-state model. Our result also highlights the role of both CT and LE diabatic states and demonstrates that consideration of the lowest adiabatic states of each spin multiplicity can be sufficient for efficient RISC.

While the model suggests that non-adiabatic coupling is not necessary for efficient RISC, it does not preclude its relevance. It is plausible that coherent mixing of $T_{1}$ and $T_{2}$ states enhances the RISC rate in the vicinity of conical intersections or avoided crossings, where the $T_{1}-T_{2}$ gap is very small. Another possibility for importance of molecular vibrations is their contribution through non-Condon effects. Within the Condon approximation all the matrix elements in Eq. 7 are constant and independent of the molecular geometry. However, molecular vibrations can lead to large variations in instantaneous couplings, effectively modulating both RISC and fluorescence. In this case, the rates depend only on nuclear positions, but not momenta, which is also consistent with the proposed conformational dependence $^{13}$ of TADF efficiency. Nevertheless, it is important to stress that neither non-adiabatic nor non-Condon effects are necessary for efficient TADF from the perspective of the four-state model.

\section{Results}

\section{Parameter space exploration}

The four-state model depends on five parameters which determine the relative energetics and character of the first two excited states within singlet and triplet manifolds. We will focus on three quantities predicted by the model, which can be optimized to boost the overall TADF efficiency. First, the singlettriplet gap $\Delta E_{S T}$ (Eq. 18) needs to be minimized to assure significant thermally-induced population of 
vibrational states of the $T_{1}$ electronic state, which are isoenergetic with the $S_{1}$ state. Second, in order to maximize the fluorescence rate, the transition dipole moment between $S_{1}$ and $S_{0}$ states needs to be as large as possible. While this quantity cannot be calculated within the four-state model without introducing the one-electron basis explicitly, we note that quantum yield correlates with the LE character of the $S_{1}$ state, which is assumed to be bright. Therefore, we consider the quantity $S_{1}^{L E}=\left|\left\langle{ }^{1} L E \mid S_{1}\right\rangle\right|^{2}$ as a proxy for fluorescence efficiency. Finally, appreciable spin-orbit coupling is a prerequisite for fast RISC even in the small $\Delta E_{S T}$ limit. The SO coupling cannot be directly calculated from the model; however, according to El-Sayed's rule it is only non-vanishing between states with substantially different electronic character. Therefore, as the proxy for efficient SO we use the unsigned difference between CT contributions in the $\mathrm{S}_{1}$ and $\mathrm{T}_{1}$ states: $\triangle C T=\left.||\left\langle{ }^{1} C T \mid S_{1}\right\rangle\right|^{2}-\left|\left\langle{ }^{3} C T \mid T_{1}\right\rangle\right|^{2} \mid$.

The analytical expressions for $\Delta E_{S T}, S_{1}^{L E}$, and $\Delta C T$ can be obtained directly by diagonalizing the model Hamiltonians in Eqs. 16 and 17; however, their dependence on the parameters is rather complicated (compare with states' coefficients in the SI). To better understand how manipulating different parameters can lead to changes in the TADF efficiency we performed a Monte Carlo sampling of the parameter space. To this end, we made some a priori assumptions about the relevant ranges of values (see SI). We sampled random $10^{6}$ points from the parameter space and calculated the corresponding $\Delta E_{S T}, S_{1}^{L E}$, and $\Delta C T$ values. Histograms of these quantities are presented in Figure 2.
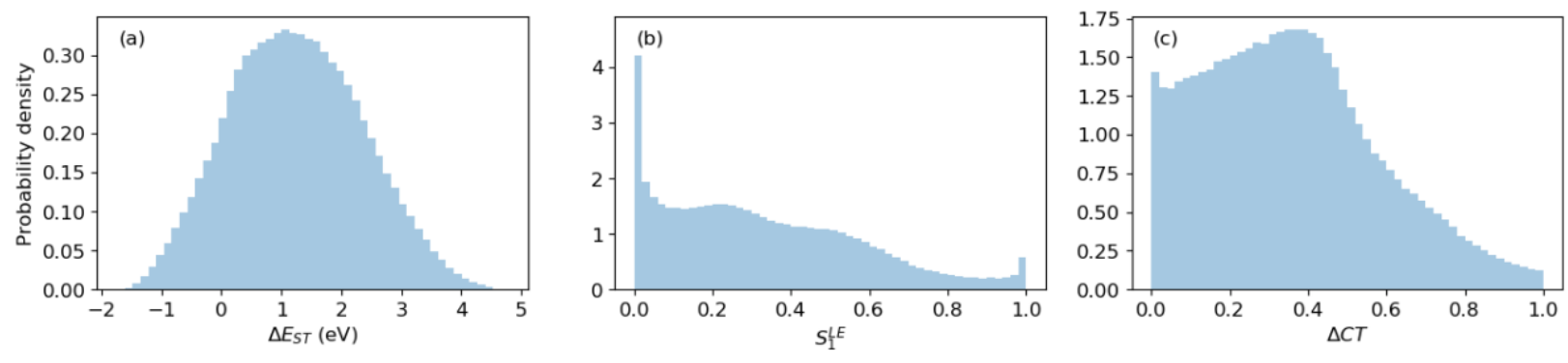
Figure 2 Distributions of $\Delta E_{S T}, S_{1}^{L E}$, and $\Delta C T$ calculated for parameters obtained from a random sampling of the parameter space.

The distribution of singlet-triplet gaps (Figure 2a) resembles a bell-shaped curve, which reflects the complex dependence on several uniformly distributed parameters (see Eq. 18). The average of $\Delta E_{S T}$ is $1.25 \mathrm{eV}$ and the standard deviation is $1.10 \mathrm{eV}$. This is in stark difference with the two-state model for which $\Delta E_{S T}=2 K_{C T}$. An interesting feature of the distribution is that there is a significant number of points with $\Delta E_{S T}<0$. This appears as perhaps an unphysical result, because negative singlet-triplet gaps are normally not observed in practice. However, based on constrained DFT calculations, Difley et al. have found exciplex systems that exhibit negative gaps ${ }^{66}$, which was explained by the kinetic exchange effect which stabilizes singlet states. Recently Olivier et al. ${ }^{51}$ also reported negative $\Delta E_{S T}$ resulting from stronger stabilization of the singlet state by the polarizable environment. Neither of these mechanisms is operational in our four-state model, which could mean that we are sampling the regions of the parameter space which are unphysical or exceedingly difficult to realize in real molecules. Nevertheless, this is not a problem of the model itself and general conclusion can still be drawn from these results. In particular, it is clear that relatively many combinations of parameters can lead to small singlet-triplet gaps, which is beneficial for fast RISC in TADF molecules.

The distribution of $S_{1}^{L E}$ (Figure $2 \mathrm{~b}$ ) shows that the first singlet excited state is usually dominated by the CT character $\left(S_{1}^{L E}<0.5\right)$. This is the case for $75 \%$ of the sampled parameter vectors and corresponds well with the experimental observations about TADF emitters. Nevertheless, the figure shows also that there are many parameter combinations which yield a significant component of the LE state in $S_{1}$, which should lead to appreciable fluorescence efficiency. Figure $2 c$ illustrates how likely it is to find parameters that lead to $S_{1}$ and $T_{1}$ states that differ in their electronic character. The maximum of the distribution is at 0.4 , and more often than not these two states are appreciably different. This is again in line with many reports 
on TADF emitters, where the lowest triplet state is found to have a substantial LE component. This in turn opens up a possibility for significant spin-orbit coupling, which would be otherwise prevented by ElSayed's rule.

To gain more insight into which parameters control TADF efficiency, we filtered the data and retained only 17706 out $10^{6}$ solutions that are likely to represent efficient TADF emitters (see SI for details). This filtering naturally selects parameter values that are beneficial for TADF and rejects parameter values that are harmful. The resulting distributions of parameters are shown in Figure 3. Figure 3a suggest that small energy offset between CT and LE states is beneficial for TADF; however, the distribution is rather broad with a standard deviation of approximately $0.5 \mathrm{eV}$ and either energetic ordering of states is possible. There is some preference for smaller values of $K_{C T}$ (Figure $3 \mathrm{~b}$ ), but many solutions can be found even with relatively large CT exchange integrals. The distribution of LE exchange integrals (Figure 3c) is also broad, with the mean $1 \mathrm{eV}$ and standard deviation $0.4 \mathrm{eV}$. Figure $3 \mathrm{~d}$ and Figure $3 \mathrm{e}$ show that both $t$ and $K_{X}$ have to be non-vanishing. In particular, the larger $K_{X}$ the more likely is that solutions represent efficient TADF and the distribution of hopping integrals $t$ has maximum at $\pm 1 \mathrm{eV}$. While all the parameters were uncorrelated in the original set, filtering is expected to introduce some correlations, and revealing them should provide guidelines for parameter combinations that yield efficient TADF activity. First, we note that while the signs of $t$ and $K_{X}$ were random and uncorrelated in the original set, now all these parameters are of the same sign; therefore, this condition appears as a prerequisite for efficient TADF. Note that flipping the phase of either $\phi_{L}$ or $\phi_{L+1}$ orbital will change the sign of both parameters, so only non-trivial changes in the orbital shape can influence the relative sign. Based on the above observations, the most critical design parameters for fast TADF are the magnitude and relative sign of $t$ and $K_{x_{*}}$. The fact that two heretofore unrecognized parameters are more important than $K_{C T}$ - which had been previously thought to dominate TADF rates - suggests that some previous design efforts may have succeeded partly 
by serendipity. For example, rotation of a dihedral angle will typically change $K_{C T}$ (which was thought to be most important) and also the magnitude and sign of $t$ and $K_{X}$ (which are typically even more important).
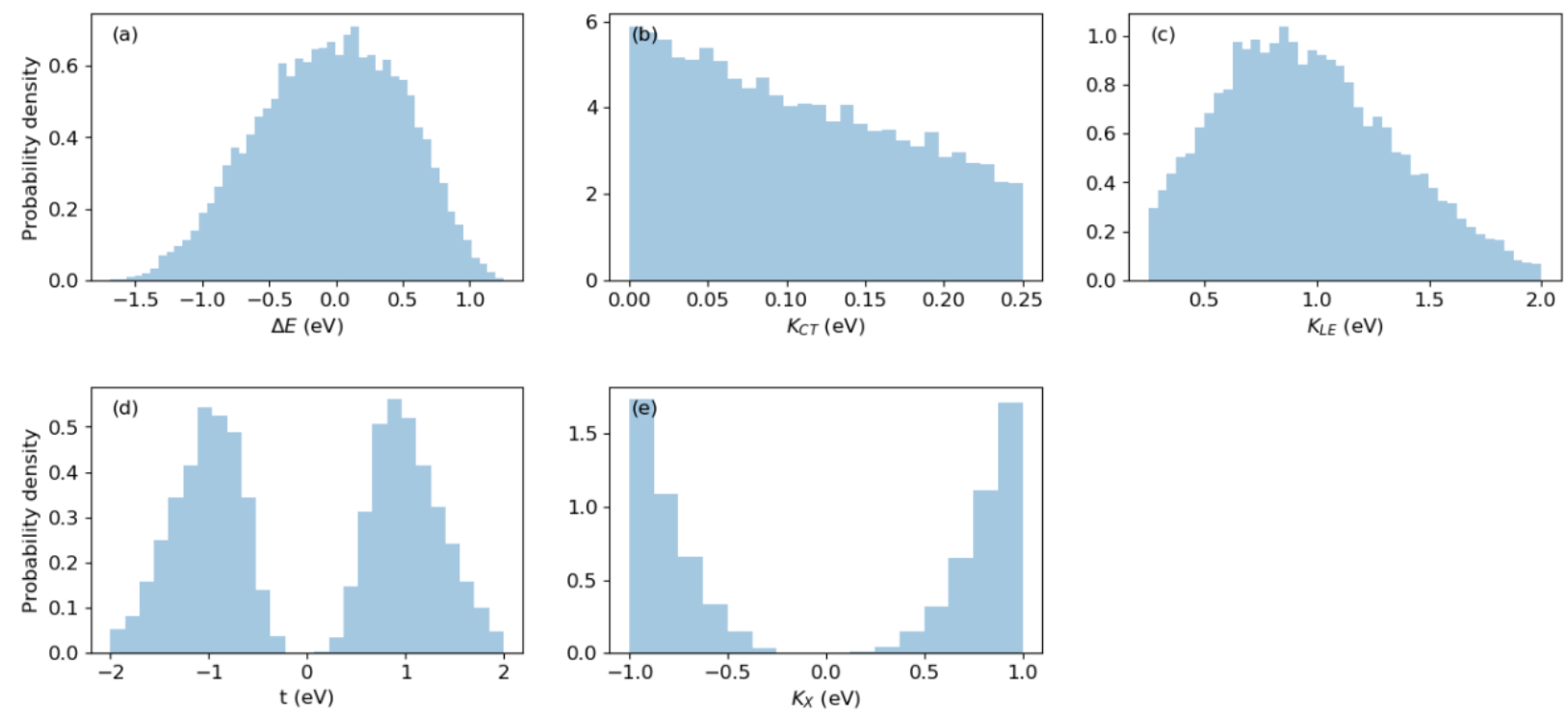

Figure 3 Histograms of parameters of the model Hamiltonian after filtering of the data.

To extract more nuanced correlations, we performed a principal component analysis (PCA) of the covariance matrix. The detailed analysis is deferred to the SI, but the main conclusion is that small values of $t-K_{X}$ and to lesser extent moderately negative $\Delta E-K_{L E}$ are necessary for efficient TADF activity. The analysis of analytical solutions of the four-state model sheds some light on the importance of $t-K_{X}$ and $\Delta E-K_{L E}$. The energy of the $\mathrm{S}_{1}$ state is stabilized by $\left|t+K_{X}\right|$ while $\mathrm{T}_{1}$ is stabilized by $\left|t-K_{X}\right|$, so small values of the latter parameter lead to shrinking of the singlet-triplet gap, which is always beneficial for TADF efficiency. Small $\left|t-K_{X}\right|$ also favors stronger LE character of the $\mathrm{T}_{1}$ state relative to $\mathrm{S}_{1}$ and consequently leads to larger $\Delta C T$ values. The energies of the ${ }^{3} L E$ and ${ }^{3} C T$ states are $\Delta E-K_{L E}$ and $-K_{C T}$, respectively. Therefore, for $\mathrm{LE}$ to be the dominant component in $\mathrm{T}_{1}$, the condition $\Delta E-K_{L E}<-K_{C T}$ must hold. At the same time, the energy gap between these states cannot be too large to allow effective 
mixing, which explains why moderately negative values of $\Delta E-K_{L E}$ are beneficial for efficient TADF. By a similar argument, appreciable $S_{1}^{L E}$ and efficient fluorescence requires that $\Delta E+K_{L E}$ is not much larger than $K_{C T}$ on the scale of the coupling element $t+K_{X}$. This suggests that, contrary to the prediction of the conventional two-state model, minimization of $K_{C T}$ is not necessarily the best strategy for maximizing TADF efficiency.

\section{Conformation space exploration}

In order to apply the design principles from the four-state model, it would be ideal to explore the parameter space of $\Delta E, t, K_{X}, K_{C T}$ and $K_{L E}$ in candidate TADF molecules. However, this is challenging for two reasons. First, the parametrization of the four-state Hamiltonian from $a b$ initio calculations requires explicit construction of the diabatic states (CT1, CT2, LE1 and LE2) and accurate evaluation of the related energies and couplings. Although the latter could be achieved through methods such as constrained $\mathrm{DFT}^{67-70}$ or $\Delta \mathrm{SCF}^{71,72}$, it is not straightforward how to define the relevant states in these frameworks. Second, optimization in the chemical space is difficult due to its discreteness. While carrying out such optimization would be part of the long-term goal that leads to new emitters, we choose at this point to explore parameter space by varying the conformation of a single donor-acceptor system (i.e. the distance and relative orientation between the donor and the acceptor moieties), which has the practical benefit of being continuous.

Our model system is based on a donor-acceptor exciplex with a TPA (triphenylamine) donor and a TRZ (2,4,6-triphenyl-1,3,5-triazine) acceptor, both of which are common moieties in TADF design ${ }^{6,14,18,29,73}$. The exciplex is known to possess intermolecular charge-transfer (CT) excited states and great conformational flexibility ${ }^{19}$. The lowest excited states have a dominant CT character due to the fact that LE states are highlying excited states in a typical exciplex. Meanwhile, according to the four-state model, we want the $T_{1}$ and $\mathrm{S}_{1}$ states of the exciplex to have a mixed CT/LE character. Therefore, we make two modifications to 
the TPA/TRZ exciplex. First, we link an extra donor CZ (carbazole) to the TRZ group to form CZ-TRZ. CZ-TRZ is a well-known fluorophore and has been used as a parent compound for several high-efficiency deepblue TADF materials ${ }^{17,21}$. Using CZ-TRZ as the acceptor in the exciplex introduces low-lying excited states with LE character, while excited states with CT character are due to excitations between TRZ and TPA moieties. Second, we add chlorine groups to TPA to form TPA-Cl. This further tunes the energetics of the CT excites states so that lowest CT and LE states are close enough, which corresponds to a small $\Delta E$ parameter in the four-state model. The final structures of TPA-Cl (donor) and CZ-TRZ (acceptor) are shown in Figure 4.
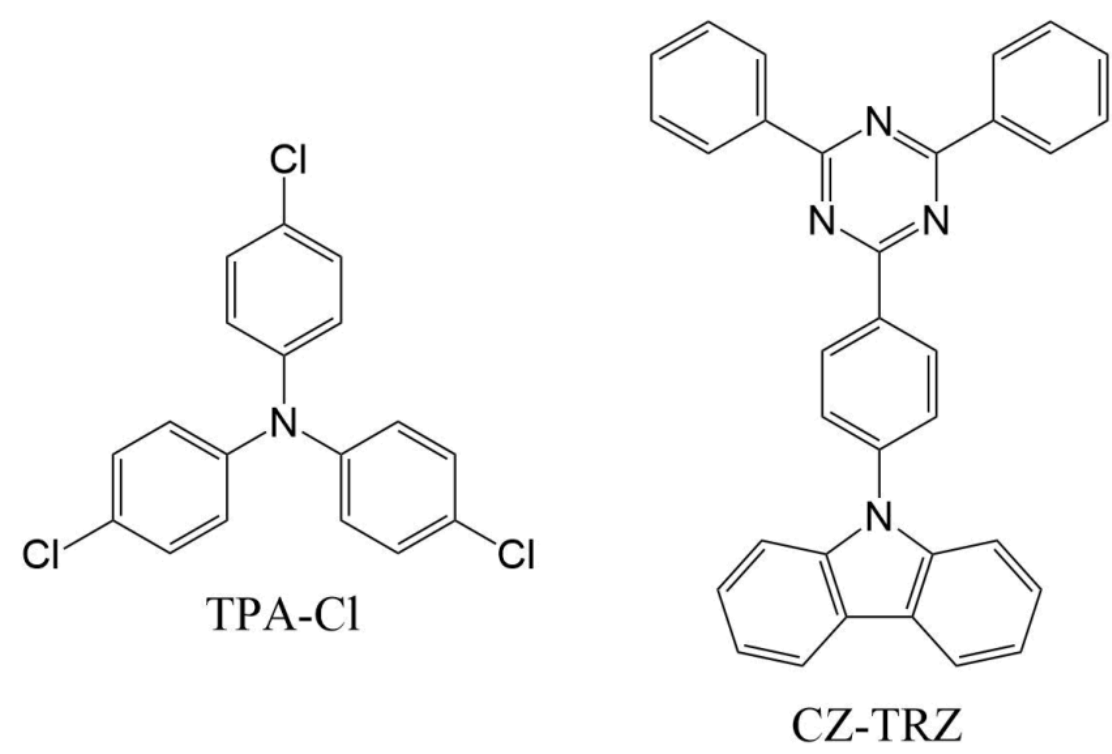

Figure 4 Structures of TPA-Cl (donor) and CZ-TRZ (acceptor).

Arbitrary conformations were generated as snapshots of molecular dynamics (MD) simulations, using the OPLS-aa force field. ${ }^{74}$ In order to obtain exhaustive sampling of the conformation space, we found it necessary to put the dimer under pressure and reduce the van der Waals parameters for the monomers. Further details can be found in the SI. As a result, the conformational space we explore is significantly different than the thermally accessible conformation space. To be clear, this is intentional: the aim of the 
calculations is to ascertain whether some configuration (which may or may not be thermally accessible for this donor and acceptor) could display dramatically accelerated TADF. If so, this observation would provide strong evidence that the four-state model is actually relevant for TADF in real molecules. The excited states in the selected conformations were computed in the framework of TD-DFT, using the B3LYP exchange-correlation functional ${ }^{75,76}$ and the $6-31+\mathrm{G}^{*}$ basis set. ${ }^{77-81}$ It is well known ${ }^{82,83}$ that standard hybrid functionals, such as B3LYP, give inadequate descriptions of CT states. Although the use of optimallytuned range-separated hybrids ${ }^{23,24}$ has been found to be a practical solution that works well for TADF emitters, the goal was not to make quantitative predictions about individual conformers, which may or may not be possible to synthesize in the laboratory, but to show that the TADF rate of a given system can undergo substantial improvements as parameters like $t$ and $K_{X}$ vary with conformation. Hence, it was desirable to choose a functional that could reproduce qualitative trends at a minimal computational cost.

Given the TD-DFT results, the rate of prompt fluorescence was estimated using the Einstein coefficient ${ }^{84}$

$$
k_{\mathrm{F}}=\frac{e^{2} \omega_{10}^{2}}{2 \pi \varepsilon_{0} m c^{3}} f_{10}
$$

where $e$ and $m$ are the electron charge and mass, respectively; $c$ is the speed of light; and $\omega_{10}$ and $f_{10}$ are the $S_{1}-S_{0}$ transition frequency and oscillator strength, respectively. The TADF rate was estimated using the formula ${ }^{12}$

$$
\begin{gathered}
k_{\mathrm{TADF}}=\frac{k_{\mathrm{F}}\left[S_{1}\right]}{\left[S_{1}\right]+\left[T_{1}\right]}=\frac{k_{\mathrm{F}}}{1+K} \\
K \equiv \frac{\left[T_{1}\right]}{\left[S_{1}\right]}=3 \exp \left(\frac{\Delta E_{S T}}{k_{\mathrm{B}} T}\right)
\end{gathered}
$$

where $k_{B}$ is the Boltzmann constant, and the temperature $T$ was taken to be $298 \mathrm{~K}$. The last formula assumes that the excited state populations have thermalized and that only the $S_{1}$ and the $T_{1}$ states are populated to a significant extent. Since TADF timescales are on the order of microseconds, the first 
assumption would hold, provided that there exists an efficient ISC pathway. Meanwhile, the second assumption should be valid in most conformations of interest. Among the conformers that exhibited the largest oscillator strengths $\left(>10^{-3}\right)$ and the smallest $\Delta E_{S T}(<100 \mathrm{meV})$, the $T_{2}$ state was almost always higher in energy than the $S_{1}$ state. If the $T_{2}$ state were lower in energy than the $S_{1}$ state, then the $T_{2}$ state should be included in the denominator, and such cases can be found in the SI. However, the correction was neglected in the main discussion, because taking the $T_{2}$ state into consideration did not alter the statistics in a meaningful way. In general, the formula provides an upper bound to the TADF rate, since RISC might not be fast enough to replenish the $S_{1}$ state.

The results have been summarized in Figure 5, and the numerical output on the most relevant conformers has been provided in the SI. Figure 5a is a histogram of the TADF lifetimes. It is clear that the DFT optimized geometry is not the optimal conformation in terms of TADF. Since there was a selection bias towards high TADF rates, the distribution is skewed, but a significant number of conformations were found to exhibit higher TADF rates than the DFT optimized geometry. A TADF lifetime as short as $2.0 \mu \mathrm{s}$ (oscillator strength of $1.4 \times 10^{-2}$ and $\Delta E_{S T}$ of $0.02 \mathrm{eV}$ ), an 800 -fold improvement over the DFT optimized geometry, has been obtained. Condensed phase effects were gauged at the level of linear-response conductor-like polarizable continuum model (LR-CPCM) ${ }^{85,86}$ the details of which have been provided in the Sl. Figure $5 \mathrm{~b}$ is a scatterplot of the condensed phase against the gas phase TADF rates. While LR-CPCM tended to stabilize the $S_{1}$ state, decrease $\Delta E_{S T}$, and increase the oscillator strength, the effects were insufficient to induce qualitative changes in the TADF rates. Hence, the remainder of the discussion will proceed based on the gas phase results. Finally, Figure $5 c$ is a scatterplot of the oscillator strength against the $\Delta E_{S T}$, testing the fundamental tradeoff predicted by the two-state model, in which a single variable (HOMO-LUMO overlap) determines both quantities. The correlation between the two variables is weaker than would be expected based on the conventional two-state model. ${ }^{7}$ The distribution may suggest an upper bound on the 
oscillator strength that can be achieved at a given $\Delta E_{S T}$ for this exciplex, but if so the slope is quite steep, allowing a sizable oscillator strength to coexist with a small $\Delta E_{S T}$. This is fairly strong evidence that by inducing large conformational changes, one can influence TADF through factors beyond the simple electron-hole overlap term that dominates the two-state model. One obvious explanation for this is that different conformations are probing variations in $t-K_{X}$ by varying the relative phase of these contributions.
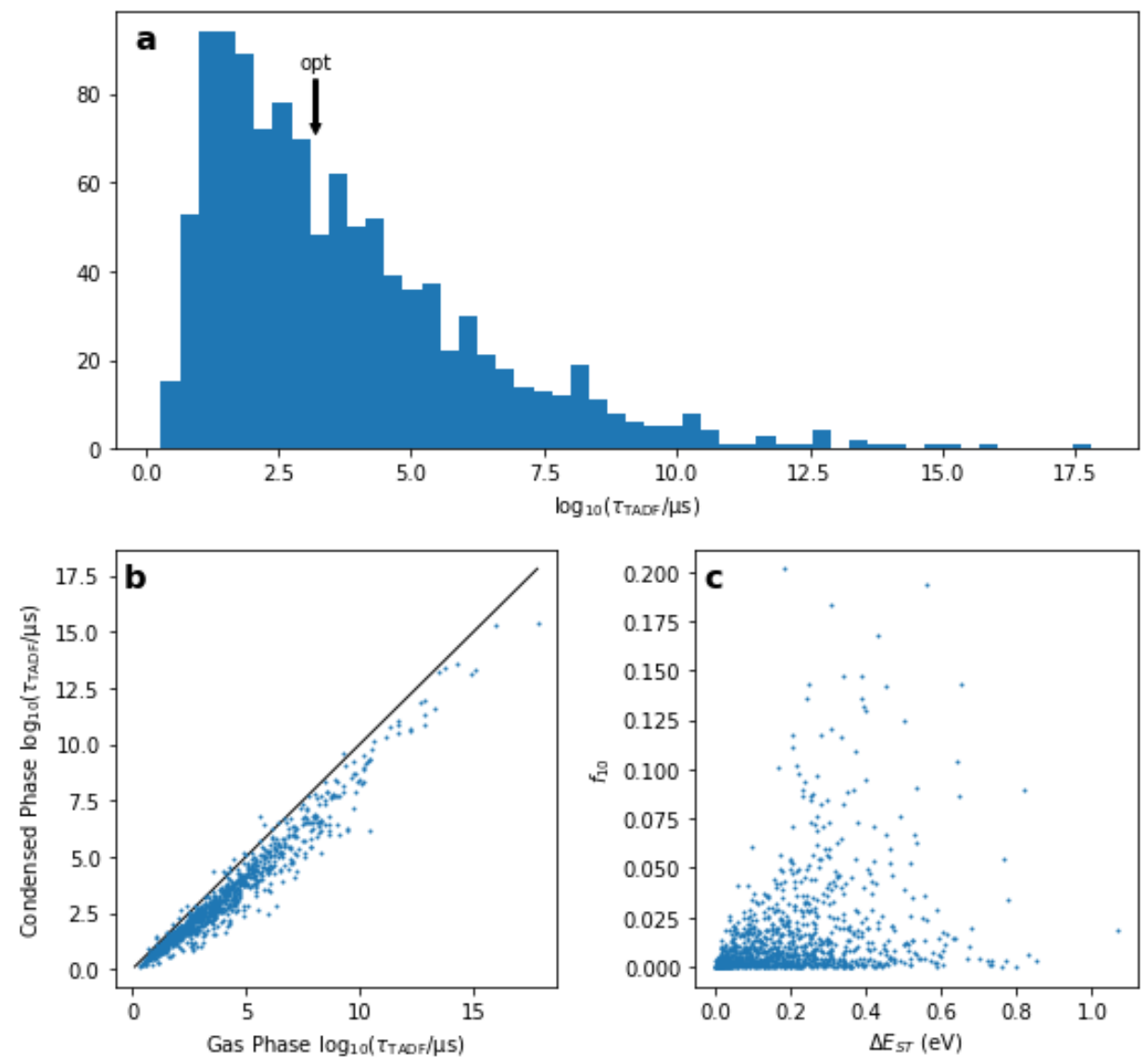

Figure 5 (a) Histogram of TADF lifetimes. (b) Scatterplot of the condensed phase against the gas phase TADF lifetimes. The solid black line has a unit slope $(y=x)$. (c) Scatterplot of the $\mathrm{S}_{1}-\mathrm{S}_{0}$ oscillator strength against $\Delta \mathrm{E}_{\mathrm{ST}}$. 
For two of the conformers that exhibited high TADF rates $\left(\tau_{\mathrm{TADF}}<10 \mu \mathrm{s}\right)$, the dominant pairs of natural transition orbitals (NTOs) ${ }^{87}$ in the $S_{1}$ and the $T_{1}$ states have been visualized in Figure 6 . Notice that the second conformer exhibits a linear conformation, with the donor moiety next to the TRZ group of the acceptor moiety, as opposed to a stacked conformation that would be expected in the equilibrium geometry. In both conformers, the spatial separation of the hole and the particle orbitals is consistent with the strong CT character of the $S_{1}$ and the $T_{1}$ excitations, but parts of the hole orbitals, as well as the particle orbital in the second conformer, can be seen delocalized onto the opposite moiety, which indicates also a non-negligible LE contribution. As a result, a substantial oscillator strength could be obtained, even when the donor and the acceptor molecules were not in a stacked conformation. Further note the differences between the NTOs of the $S_{1}$ and the $T_{1}$ states, which are most obvious in the delocalized lobes of the hole and the particle orbitals. In particular, the $\mathrm{T}_{1}$ excitation appears to have a stronger LE character than the $S_{1}$ excitation. Again, this is consistent with the predictions of the four-state model, where differential mixing of CT and LE states is possible in the singlet and triplet manifolds. We suspect that the differences between the $S_{1}$ and the $T_{1}$ states for this dimer would be even more pronounced in reality and that their apparent similarity is due to the abovementioned shortcoming of the B3LYP functional favoring CT states. Other conformers also exhibited varying degrees of CT/LE mixture in the $S_{1}$ and the $T_{1}$ states, and more examples have been provided in the SI (Figures S2-S3). A more detailed comparison of the $S_{1}$ and the $T_{1}$ states based on the Kohn-Sham orbitals can also be found in the SI. 

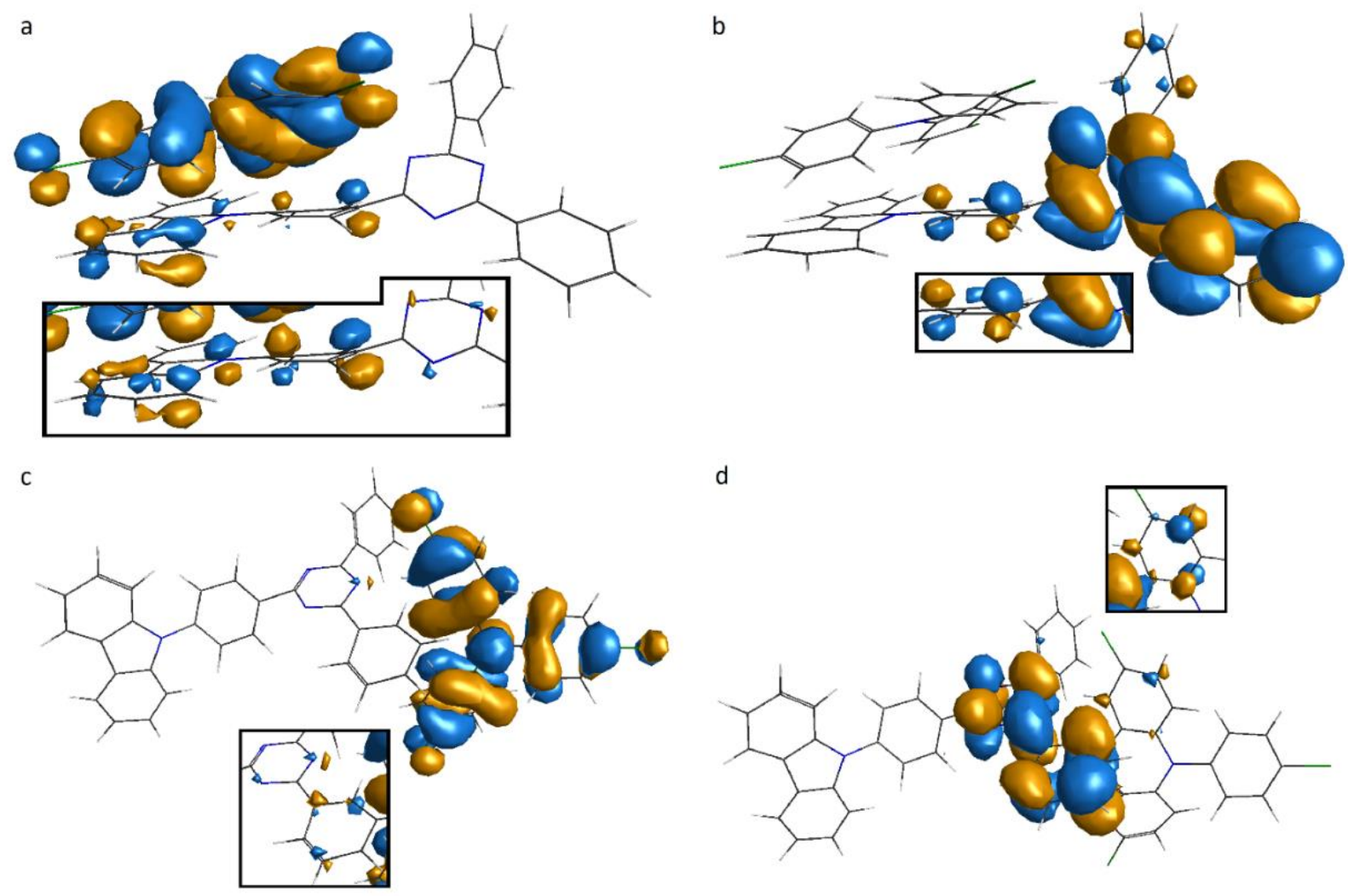

Figure $6(a, c)$ Hole and $(b, d)$ particle orbitals of the $S_{1}$ state. The insets are the corresponding NTOs of the $T_{1}$ state.

All in all, a more sophisticated computational method or an experiment could reveal most of the found conformers to be poor TADF emitters. For the purposes of this study, however, it sufficed to show that a large oscillator strength, a small $\Delta E_{S T}$, and a substantial spin-orbit coupling can coexist in the framework of a reasonable density functional. A priori, it should be no easier to optimize a set of properties in the DFT world than it is in the real world. The in silico discovery of efficient TADF via spin-orbit coupling should motivate a search for such systems in vitro.

\section{Discussion}

Turning to the ensemble statistics of the conformers, the most obvious feature is the order-of-magnitude variations in the oscillator strengths and the energy gaps, indicating strong non-Condon effects. Although 
the conformations may be unrealistic as equilibrium geometries, they are realistic as snapshots in time. Further assuming the validity of the Born-Oppenheimer approximation, the TADF rates based on the TDDFT results are just the instantaneous rates at the corresponding times along the trajectory. Hence, the results support the proposition that the instantaneous TADF rate of a system can be maximized at some non-equilibrium geometry. There might even exist a system where most accessible conformations have higher TADF rate than the equilibrium geometry. The existence of such system would help explain the experimental account of Ward and collaborators ${ }^{58}$ that TADF in a D-A-D system diminished when the motion of the donor and the acceptor moieties was hindered. Indeed, constraining the geometry would be detrimental if thermal motion gave access to an ensemble of conformations with higher TADF rates. On the other hand, it would also be possible to harness the conformational dependence by freezing an emitter in the conformation or the range of conformations that maximizes the rate. Such design strategy becomes viable in light of the four-state model's implication that the rates depend only on the nuclear positions, and not on the momenta.

Of course, there are some questions that must be answered before conformational variation can lead to the improvement of real TADF emitters. First, there is the theoretical problem of determining the structural features that facilitate TADF. In the model system, there did not appear to be a particular distance, torsion or orientation that could be conclusively associated with high TADF rates. Perhaps it was to be expected, considering the intricate interplay of several variables in the four-state model. Then, there is the experimental problem of synthesizing molecules in a specific conformation. While there have been efforts to tune the conformation of TADF emitters using steric hindrance, ${ }^{17,58}$ there does not appear to be an established method or guideline to date. Nevertheless, conformational variation introduces a new dimension over which TADF emitters can be optimized. Further theoretical and experimental efforts 
should be directed to elucidating the structural features that facilitate TADF and the synthetic techniques of enforcing these structures on real molecular systems.

\section{Conclusions}

We have proposed a simple four-state model which explains the key prerequisites for efficient thermally activated delayed fluorescence in organic donor-acceptor systems. The four spin-mixed diabatic states constitute the conceptual basis to discuss the electronic structure of TADF emitters. We show that the resulting lowest-lying adiabatic triplet $\left(T_{1}\right)$ and singlet $\left(S_{1}\right)$ excited states can have the necessary characteristics to assure efficient TADF. These characteristics are small singlet-triplet gap, different electronic character of $S_{1}$ and $T_{1}$, and significant component of the local excitation in $S_{1}$. The first two conditions enable fast reverse intersystem crossing, while the last one enables appreciable quantum yields. The model suggests that non-adiabatic effects are not necessary for efficient TADF; however, it does not rule out their contribution completely.

Monte Carlo sampling reveals that the conditions for efficient TADF are relatively easy to satisfy in the parameter space; however, it does not mean that it is equally easy to realize them in real molecular systems. The statistical analysis of the data filtered for TADF activity reveals that a close mutual alignment of some pairs of parameters of the model is a prerequisite for TADF, while their magnitude fine-tunes its efficiency. This observation provides new design principles for optimization of organic emitters, which should aim at satisfying the parameter alignment criteria.

Establishing the structure-property relationships between different molecular architectures and model parameters should be the next step in the exploration of the four-state model, which would lead eventually to the exploration of the chemical space. In the meantime, by means of combined molecular dynamics and TD-DFT simulations for a model exciplex systems, we show that significant increase of TADF 
efficiency can be also obtained by exploration of the conformation space. This supports the vibronic mechanism for TADF, in which the non-Condon effects modulate the instantaneous TADF rates and maximize them for some non-equilibrium conformations. Additionally, our study suggests that optimization of condensed-phase molecular conformations, e.g. through controlled exploitation of steric hindrance or application of pressure may be a practical design strategy for improved OLED materials.

\section{Acknowledgements}

This work was funded by a grant from the US Department of Energy, Basic Energy Sciences (BES ER46474).

\section{Supporting Information}

Approximations behind the four-state Hamiltonian; analytic expressions for eigenvalues and eigenvectors of the four-state Hamiltonian; details of the Monte Carlo sampling, principal component analysis, MD simulations; images of natural transition orbitals; analysis of the orbital transitions; further details on the TADF rate expression; numerical outputs of TD-DFT calculations; details and results of the LR-CPCM/TDDFT calculations.

\section{References}

(1) Perrin, F. Fluorescence of Solutions. Ann. Phys. 1929, 10 (12), 169-275.

(2) Parker, C. A.; Hatchard, C. G. Triplet-Singlet Emission in Fluid Solutions. Phosphorescence of Eosin. Trans. Faraday Soc. 1961.

(3) Endo, A.; Ogasawara, M.; Takahashi, A.; Kato, Y.; Adachi, C. Thermally Activated Delayed Fluorescence (TADF) from Sn4+- Porphyrin Complexes and Their Application to Organic Light Emitting Diodes - Novel Pathway for High Efficiency Electroluminescence. Proc. Int. Meet. Inf. Disp. 2008, 8. 
(4) Endo, A.; Sato, K.; Yoshimura, K.; Kai, T.; Kawada, A.; Miyazaki, H.; Adachi, C. Efficient UpConversion of Triplet Excitons into a Singlet State and Its Application for Organic Light Emitting Diodes. Appl. Phys. Lett. 2011, 98 (8), 083302.

(5) Goushi, K.; Yoshida, K.; Sato, K.; Adachi, C. Organic Light-Emitting Diodes Employing Efficient Reverse Intersystem Crossing for Triplet-to-Singlet State Conversion. Nat. Photonics 2012, 6 (4), 253-258.

(6) Tanaka, H.; Shizu, K.; Miyazaki, H.; Adachi, C. Efficient Green Thermally Activated Delayed Fluorescence (TADF) from a Phenoxazine-Triphenyltriazine (PXZ-TRZ) Derivative. Chem. Commun. 2012, 48 (93), 11392-11394.

(7) Uoyama, H.; Goushi, K.; Shizu, K.; Nomura, H.; Adachi, C. Highly Efficient Organic Light-Emitting Diodes from Delayed Fluorescence. Nature 2012, 492 (7428), 234-238.

(8) Zhang, Q.; Li, J.; Shizu, K.; Huang, S.; Hirata, S.; Miyazaki, H.; Adachi, C. Design of Efficient Thermally Activated Delayed Fluorescence Materials for Pure Blue Organic Light Emitting Diodes. J. Am. Chem. Soc. 2012, 134 (36), 14706-14709.

(9) Lee, J.; Shizu, K.; Tanaka, H.; Nomura, H.; Yasuda, T.; Adachi, C. Oxadiazole- and Triazole-Based Highly-Efficient Thermally Activated Delayed Fluorescence Emitters for Organic Light-Emitting Diodes. J. Mater. Chem. C 2013, 1 (30), 4599.

(10) Dias, F. B.; Bourdakos, K. N.; Jankus, V.; Moss, K. C.; Kamtekar, K. T.; Bhalla, V.; Santos, J.; Bryce, M. R.; Monkman, A. P. Triplet Harvesting with 100\% Efficiency by Way of Thermally Activated Delayed Fluorescence in Charge Transfer OLED Emitters. Adv. Mater. 2013, 25 (27), 3707-3714.

(11) Liu, Y.; Li, C.; Ren, Z.; Yan, S.; Bryce, M. R. All-Organic Thermally Activated Delayed Fluorescence Materials for Organic Light-Emitting Diodes. Nat. Rev. Mater. 2018, 3 (4), 18020. 
(12) Zhang, Q.; Li, B.; Huang, S.; Nomura, H.; Tanaka, H.; Adachi, C. Efficient Blue Organic Light-Emitting Diodes Employing Thermally Activated Delayed Fluorescence. Nat. Photonics 2014, 8 (4), 326-332.

(13) Hirata, S.; Sakai, Y.; Masui, K.; Tanaka, H.; Lee, S. Y.; Nomura, H.; Nakamura, N.; Yasumatsu, M.; Nakanotani, H.; Zhang, Q.; et al. Highly Efficient Blue Electroluminescence Based on Thermally Activated Delayed Fluorescence. Nat. Mater. 2015, 14 (3), 330-336.

(14) Kawasumi, K.; Wu, T.; Zhu, T.; Chae, H. S.; Van Voorhis, T.; Baldo, M. A.; Swager, T. M. Thermally Activated Delayed Fluorescence Materials Based on Homoconjugation Effect of Donor-Acceptor Triptycenes. J. Am. Chem. Soc. 2015, 137 (37), 11908-11911.

(15) Nobuyasu, R. S.; Ren, Z.; Griffiths, G. C.; Batsanov, A. S.; Data, P.; Yan, S.; Monkman, A. P.; Bryce, M. R.; Dias, F. B. Rational Design of TADF Polymers Using a Donor-Acceptor Monomer with Enhanced TADF Efficiency Induced by the Energy Alignment of Charge Transfer and Local Triplet Excited States. Adv. Opt. Mater. 2016, 4 (4), 597-607.

(16) Liu, Y.; Xie, G.; Wu, K.; Luo, Z.; Zhou, T.; Zeng, X.; Yu, J.; Gong, S.; Yang, C. Boosting Reverse Intersystem Crossing by Increasing Donors in Triarylboron/Phenoxazine Hybrids: TADF Emitters for High-Performance Solution-Processed OLEDs. J. Mater. Chem. C 2016, 4 (20), 4402-4407.

(17) Huang, W.; Einzinger, M.; Zhu, T.; Chae, H. S.; Jeon, S.; Ihn, S.-G. G.; Sim, M.; Kim, S.; Su, M.; Teverovskiy, G.; et al. Molecular Design of Deep Blue Thermally Activated Delayed Fluorescence Materials Employing a Homoconjugative Triptycene Scaffold and Dihedral Angle Tuning. Chem. Mater. 2018, 30 (5), 1462-1466.

(18) Wong, M. Y.; Zysman-Colman, E. Purely Organic Thermally Activated Delayed Fluorescence Materials for Organic Light-Emitting Diodes. Adv. Mater. 2017, 29 (22), 1605444.

(19) Sarma, M.; Wong, K.-T. Exciplex: An Intermolecular Charge-Transfer Approach for TADF. ACS Appl. 
Mater. Interfaces 2018, 10 (23), 19279-19304.

(20) Dos Santos, P. L.; Ward, J. S.; Bryce, M. R.; Monkman, A. P. Using Guest-Host Interactions to Optimize the Efficiency of TADF OLEDs. J. Phys. Chem. Lett. 2016, 7 (17), 3341-3346.

(21) Cui, L.-S.; Nomura, H.; Geng, Y.; Kim, J. U.; Nakanotani, H.; Adachi, C. Controlling Singlet-Triplet Energy Splitting for Deep-Blue Thermally Activated Delayed Fluorescence Emitters. Angew. Chemie Int. Ed. 2017, 56 (6), 1571-1575.

(22) Huang, S.; Zhang, Q.; Shiota, Y.; Nakagawa, T.; Kuwabara, K.; Yoshizawa, K.; Adachi, C. Computational Prediction for Singlet- and Triplet-Transition Energies of Charge-Transfer Compounds. J. Chem. Theory Comput. 2013, 9 (9), 3872-3877.

(23) Penfold, T. J. On Predicting the Excited-State Properties of Thermally Activated Delayed Fluorescence Emitters. J. Phys. Chem. C 2015, 119 (24), 13535-13544.

(24) Sun, H.; Zhong, C.; Brédas, J. L. Reliable Prediction with Tuned Range-Separated Functionals of the Singlet-Triplet Gap in Organic Emitters for Thermally Activated Delayed Fluorescence. J. Chem. Theory Comput. 2015, 11 (8), 3851-3858.

(25) Hait, D.; Zhu, T.; McMahon, D. P.; Van Voorhis, T. Prediction of Excited-State Energies and SingletTriplet Gaps of Charge-Transfer States Using a Restricted Open-Shell Kohn-Sham Approach. J. Chem. Theory Comput. 2016, 12 (7), 3353-3359.

(26) Valchanov, G.; Ivanova, A.; Tadjer, A.; Chercka, D.; Baumgarten, M. Understanding the Fluorescence of TADF Light-Emitting Dyes. J. Phys. Chem. A 2016, 120 (35), 6944-6955.

(27) Moral, M.; Muccioli, L.; Son, W. J.; Olivier, Y.; Sancho-Garcia, J. C. Theoretical Rationalization of the Singlet-Triplet Gap in Oleds Materials: Impact of Charge-Transfer Character. J. Chem. Theory Comput. 2015, $11(1), 168-177$. 
Chen, T.; Zheng, L.; Yuan, J.; An, Z.; Chen, R.; Tao, Y.; Li, H.; Xie, X.; Huang, W. Understanding the Control of Singlet-Triplet Splitting for Organic Exciton Manipulating: A Combined Theoretical and Experimental Approach. Sci. Rep. 2015, 5 (January), 10923.

(29) Gómez-Bombarelli, R.; Aguilera-Iparraguirre, J.; Hirzel, T. D.; Duvenaud, D.; Maclaurin, D.; BloodForsythe, M. A.; Chae, H. S.; Einzinger, M.; Ha, D. G.; Wu, T.; et al. Design of Efficient Molecular Organic Light-Emitting Diodes by a High-Throughput Virtual Screening and Experimental Approach. Nat. Mater. 2016, 15 (10), 1120-1127.

(30) Shu, Y.; Levine, B. G. Simulated Evolution of Fluorophores for Light Emitting Diodes. J. Chem. Phys. 2015, 142 (10).

(31) Rupakheti, C.; Al-Saadon, R.; Zhang, Y.; Virshup, A. M.; Zhang, P.; Yang, W.; Beratan, D. N. Diverse Optimal Molecular Libraries for Organic Light-Emitting Diodes. J. Chem. Theory Comput. 2016, 12 (4), 1942-1952.

Dias, F. B.; Santos, J.; Graves, D. R.; Data, P.; Nobuyasu, R. S.; Fox, M. A.; Batsanov, A. S.; Palmeira, T.; Berberan-Santos, M. N.; Bryce, M. R.; et al. The Role of Local Triplet Excited States and D-A Relative Orientation in Thermally Activated Delayed Fluorescence: Photophysics and Devices. Adv. Sci. 2016, 3 (12), 1-10.

Hosokai, T.; Noda, H.; Nakanotani, H.; Nawata, T.; Nakayama, Y.; Matsuzaki, H.; Adachi, C. SolventDependent Investigation of Carbazole Benzonitrile Derivatives: Does the LE3-CT1 Energy Gap Facilitate Thermally Activated Delayed Fluorescence? J. Photonics Energy 2018, 8 (03), 1.

(34) Gan, L.; Gao, K.; Cai, X.; Chen, D.; Su, S.-J. Achieving Efficient Triplet Exciton Utilization with Large $\Delta$ E ST and Nonobvious Delayed Fluorescence by Adjusting Excited State Energy Levels. J. Phys. Chem. Lett. 2018, 9 (16), 4725-4731. 
(35) Lee, K.; Kim, D. Local-Excitation versus Charge-Transfer Characters in the Triplet State: Theoretical Insight into the Singlet-Triplet Energy Differences of Carbazolyl-Phthalonitrile-Based Thermally Activated Delayed Fluorescence Materials. J. Phys. Chem. C 2016, 120 (49), 28330-28336.

(36)

Samanta, P. K.; Kim, D.; Coropceanu, V.; Brédas, J. L. Up-Conversion Intersystem Crossing Rates in Organic Emitters for Thermally Activated Delayed Fluorescence: Impact of the Nature of Singlet vs Triplet Excited States. J. Am. Chem. Soc. 2017, 139 (11), 4042-4051.

Kim, D. A Theoretical Understanding of the Energy Difference between Singlet and Triplet States of Oligoacene Molecules. Int. J. Quantum Chem. 2016, 116 (8), 651-655.

(38) Kim, D. Effects of Intermolecular Interactions on the Singlet-Triplet Energy Difference: A Theoretical Study of the Formation of Excimers in Acene Molecules. J. Phys. Chem. C 2015, 119 (22), 12690-12697.

(39) Gao, Y.; Su, T.; Zhao, L.; Geng, Y.; Wu, Y.; Zhang, M.; Su, Z. M. How Does a Little Difference in Structure Determine Whether Molecules Have Thermally Activated Delayed Fluorescence Characteristic or Not? Org. Electron. physics, Mater. Appl. 2017, 50, 70-76.

(40) Duan, Y.-C.; Wen, L.-L.; Gao, Y.; Wu, Y.; Zhao, L.; Geng, Y.; Shan, G.-G.; Zhang, M.; Su, Z.-M. Fluorescence, Phosphorescence, or Delayed Fluorescence? - A Theoretical Exploration on the Reason Why a Series of Similar Organic Molecules Exhibit Different Luminescence Types. J. Phys. Chem. C 2018, acs.jpcc.8b06533.

(41) Chen, X.-K. K.; Zhang, S.-F. F.; Fan, J.-X. X.; Ren, A.-M. M. Nature of Highly Efficient Thermally Activated Delayed Fluorescence in Organic Light-Emitting Diode Emitters: Nonadiabatic Effect between Excited States. J. Phys. Chem. C 2015, 119 (18), 9728-9733.

(42) Gibson, J.; Monkman, A. P.; Penfold, T. J. The Importance of Vibronic Coupling for Efficient Reverse 
Intersystem Crossing in Thermally Activated Delayed Fluorescence Molecules. ChemPhysChem 2016, 17 (19), 2956-2961.

(43) Etherington, M. K.; Gibson, J.; Higginbotham, H. F.; Penfold, T. J.; Monkman, A. P. Revealing the Spin-Vibronic Coupling Mechanism of Thermally Activated Delayed Fluorescence. Nat. Commun. 2016, 7, 13680.

(44) Marian, C. M. Mechanism of the Triplet-to-Singlet Upconversion in the Assistant Dopant ACRXTN. J. Phys. Chem. C 2016, 120 (7), 3715-3721.

(45) Lin, L.; Cai, L.; Fan, J.; Wang, C. K. Electroluminescent Mechanism of Thermally Activated Delayed Fluorescence Emitters: Conformational Effect. J. Phys. Chem. C 2018, 122 (34), 19953-19961.

(46) Sun, H.; Hu, Z.; Zhong, C.; Chen, X.; Sun, Z.; Brédas, J. L. Impact of Dielectric Constant on the SingletTriplet Gap in Thermally Activated Delayed Fluorescence Materials. J. Phys. Chem. Lett. 2017, 8 (11), 2393-2398.

Mewes, J. M. Modeling TADF in Organic Emitters Requires a Careful Consideration of the Environment and Going beyond the Franck-Condon Approximation. Phys. Chem. Chem. Phys. 2018, 20 (18), 12454-12469.

(48) Northey, T.; Stacey, J.; Penfold, T. J. The Role of Solid State Solvation on the Charge Transfer State of a Thermally Activated Delayed Fluorescence Emitter. J. Mater. Chem. C 2017, 5 (42), 1100111009.

(49) Lv, L.; Liu, K.; Yuan, K.; Zhu, Y.; Wang, Y. Thermally Activated Delayed Fluorescence Processes for $\mathrm{Cu}(\mathrm{i})$ Complexes in Solid-State: A Computational Study Using Quantitative Prediction. RSC Adv. 2018, 8 (50), 28421-28432.

(50) de Silva, P.; Van Voorhis, T. QM/MM Study of Static and Dynamic Energetic Disorder in the Emission 
Layer of an Organic Light-Emitting Diode. J. Phys. Chem. Lett. 2018, 9 (6), 1329-1334.

(51) Olivier, Y.; Yurash, B.; Muccioli, L.; Avino, G. D.; Mikhnenko, O.; Adachi, C.; D’Avino, G.; Mikhnenko, O.; Sancho-García, J. C.; Adachi, C.; et al. Nature of the Singlet and Triplet Excitations Mediating Thermally Activated Delayed Fluorescence. Phys. Rev. Mater. 2017, 075602 (7), 1-6.

(52) Olivier, Y.; Sancho-García, J. C.; Muccioli, L.; D’Avino, G.; Beljonne, D. Computational Design of Thermally Activated Delayed Fluorescence Materials: The Challenges Ahead. J. Phys. Chem. Lett. 2018, 9, acs.jpclett.8b02327.

(53) Chen, X.-K.; Kim, D.; Brédas, J.-L. Thermally Activated Delayed Fluorescence (TADF) Path toward Efficient Electroluminescence in Purely Organic Materials: Molecular Level Insight. Acc. Chem. Res. 2018, 51 (9), acs.accounts.8b00174.

(54) Penfold, T. J.; Dias, F. B.; Monkman, A. P. The Theory of Thermally Activated Delayed Fluorescence for Organic Light Emitting Diodes. Chemical Communications. Royal Society of Chemistry April 17, 2018, pp 3926-3935.

(55) Dias, F. B.; Penfold, T. J.; Monkman, A. P. Photophysics of Thermally Activated Delayed Fluorescence Molecules. Methods Appl. Fluoresc. 2017, 5 (1), 012001.

(56) El-Sayed, M. A. Spin-Orbit Coupling and the Radiationless Processes in Nitrogen Heterocyclics. J. Chem. Phys. 1963.

(57) Marian, C. M. Spin-Orbit Coupling and Intersystem Crossing in Molecules. Wiley Interdiscip. Rev. Comput. Mol. Sci. 2012, 2 (2), 187-203.

(58) Ward, J. S.; Nobuyasu, R. S.; Batsanov, A. S.; Data, P.; Monkman, A. P.; Dias, F. B.; Bryce, M. R. The Interplay of Thermally Activated Delayed Fluorescence (TADF) and Room Temperature Organic Phosphorescence in Sterically-Constrained Donor-Acceptor Charge-Transfer Molecules. Chem. 
Commun. 2016, 52 (12), 2612-2615.

(59) Yersin, H.; Mataranga-Popa, L.; Li, S. W.; Czerwieniec, R. Design Strategies for Materials Showing Thermally Activated Delayed Fluorescence and beyond: Towards the Fourth-Generation OLED Mechanism. J. Soc. Inf. Disp. 2018, 26 (4), 194-199.

(60) Chen, X.-K. K.; Tsuchiya, Y.; Ishikawa, Y.; Zhong, C.; Adachi, C.; Brédas, J.-L. L. A New Design Strategy for Efficient Thermally Activated Delayed Fluorescence Organic Emitters: From Twisted to Planar Structures. Adv. Mater. 2017, 1702767 (46), 1702767.

(61) Einzinger, M.; Zhu, T.; de Silva, P.; Belger, C.; Swager, T. M.; Van Voorhis, T.; Baldo, M. A. Shorter Exciton Lifetimes via an External Heavy-Atom Effect: Alleviating the Effects of Bimolecular Processes in Organic Light-Emitting Diodes. Adv. Mater. 2017, 29 (40).

(62) Zhang, W.; Jin, J.; Huang, Z.; Zhuang, S.; Wang, L. A New Way towards High-Efficiency Thermally Activated Delayed Fluorescence Devices via External Heavy-Atom Effect. Sci. Rep. 2016, 6 (1), 30178.

(63) Lyskov, I.; Marian, C. M. Climbing up the Ladder: Intermediate Triplet States Promote the Reverse Intersystem Crossing in the Efficient TADF Emitter ACRSA. J. Phys. Chem. C 2017, 121 (39), 2114521153.

(64) Penfold, T. J.; Gindensperger, E.; Daniel, C.; Marian, C. M. Spin-Vibronic Mechanism for Intersystem Crossing. Chem. Rev. 2018, 118 (15), 6975-7025.

(65) Gibson, J.; Penfold, T. J. Nonadiabatic Coupling Reduces the Activation Energy in Thermally Activated Delayed Fluorescence. Phys. Chem. Chem. Phys. 2017, 19 (12), 8428-8434.

(66) Difley, S.; Beljonne, D.; Voorhis, T. Van. On the Singlet-Triplet Splitting of Geminate Electron-Hole Pairs in Organic Semiconductors. J. Am. Chem. Soc. 2008, 130 (11), 3420-3427. 
(67) Wu, Q.; Van Voorhis, T. Extracting Electron Transfer Coupling Elements from Constrained Density Functional Theory. J. Chem. Phys. 2006, 125 (16), 164105.

(68) Kaduk, B.; Kowalczyk, T.; Van Voorhis, T. Constrained Density Functional Theory. Chem. Rev. 2012, $112(1), 321-370$.

(69) Wu, Q.; Van Voorhis, T. Direct Optimization Method to Study Constrained Systems within DensityFunctional Theory. Phys. Rev. A 2005, 72 (2), 024502.

(70) Wu, Q.; Van Voorhis, T. Direct Calculation of Electron Transfer Parameters through Constrained Density Functional Theory. J. Phys. Chem. A 2006, 110 (29), 9212-9218.

(71) Gilbert, A. T. B.; Besley, N. A.; Gill, P. M. W. Self-Consistent Field Calculations of Excited States Using the Maximum Overlap Method. J. Phys. Chem. A 2008, 112 (50), 13164-13171.

(72) Kowalczyk, T.; Yost, S. R.; Voorhis, T. Van. Assessment of the $\Delta$ sCF Density Functional Theory Approach for Electronic Excitations in Organic Dyes. J. Chem. Phys. 2011, 134 (5).

(73) Im, Y.; Kim, M.; Cho, Y. J.; Seo, J.-A.; Yook, K. S.; Lee, J. Y. Molecular Design Strategy of Organic Thermally Activated Delayed Fluorescence Emitters. Chem. Mater. 2017, 29 (5), 1946-1963.

(74) Jorgensen, W. L.; Maxwell, D. S.; Tirado-Rives, J. Development and Testing of the OPLS All-Atom Force Field on Conformational Energetics and Properties of Organic Liquids. J. Am. Chem. Soc. 1996, $118(45), 11225-11236$.

(75) Becke, A. D. Density-functional Thermochemistry. III. The Role of Exact Exchange. J. Chem. Phys. 1993, $98(7), 5648-5652$.

(76) Stephens, P. J.; Devlin, F. J.; Chabalowski, C. F.; Frisch, M. J. Ab Initio Calculation of Vibrational Absorption and Circular Dichroism Spectra Using Density Functional Force Fields. J. Phys. Chem. 
1994, $98(45), 11623-11627$.

(77) Hariharan, P. C.; Pople, J. A. The Influence of Polarization Functions on Molecular Orbital Hydrogenation Energies. Theor. Chim. Acta 1973, 28 (3), 213-222.

(78) Francl, M. M.; Pietro, W. J.; Hehre, W. J.; Binkley, J. S.; Gordon, M. S.; DeFrees, D. J.; Pople, J. A. Self-Consistent Molecular Orbital Methods. XXIII. A Polarization-Type Basis Set for Second-Row Elements. J. Chem. Phys. 1982, 77 (7), 3654-3665.

(79) Clark, T.; Chandrasekhar, J.; Spitznagel, G. W.; Schleyer, P. v R. Efficient Diffuse FunctionAugmented Basis Sets for Anion Calculations. III. The 3-21+G Basis Set for First-Row Elements, Lithium to Fluorine. J. Comput. Chem. 1983, 4 (3), 294-301.

(80) Krishnan, R.; Binkley, J. S.; Seeger, R.; Pople, J. A. Self-Consistent Molecular Orbital Methods. XX. A Basis Set for Correlated Wave Functions. J. Chem. Phys. 1980, 72 (1), 650-654.

(81) Gill, P. M. W.; Johnson, B. G.; Pople, J. A.; Frisch, M. J. The Performance of the Becke-Lee-Yang-Parr (B-LYP) Density Functional Theory with Various Basis Sets. Chem. Phys. Lett. 1992, 197 (4,5), 499505.

(82) Dreuw, A.; Head-Gordon, M. Failure of Time-Dependent Density Functional Theory for Long-Range Charge-Transfer Excited States: The Zincbacteriochlorin-Bacteriochlorin and BacteriochlorophyllSpheroidene Complexes. J. Am. Chem. Soc. 2004, 126 (12), 4007-4016.

(83) Dreuw, A.; Head-Gordon, M. Single-Reference Ab Initio Methods for the Calculation of Excited States of Large Molecules. Chem. Rev. 2005, 105 (11), 4009-4037.

(84) Hilborn, R. C. Einstein Coefficients, Cross Sections, f Values, Dipole Moments, and All That. Am. J. Phys. 1982, 50, 982. 
(85) You, Z. Q.; Mewes, J. M.; Dreuw, A.; Herbert, J. M. Comparison of the Marcus and Pekar Partitions in the Context of Non-Equilibrium, Polarizable-Continuum Solvation Models. J. Chem. Phys. 2015, $143(20)$.

(86) Mewes, J. M.; You, Z. Q.; Wormit, M.; Kriesche, T.; Herbert, J. M.; Dreuw, A. Experimental Benchmark Data and Systematic Evaluation of Two a Posteriori, Polarizable-Continuum Corrections for Vertical Excitation Energies in Solution. J. Phys. Chem. A 2015, 119 (21), 5446-5464.

(87) Martin, R. L. Natural Transition Orbitals. J. Chem. Phys. 2003, 118, 4775-4777. 
Extracting Design Principles for Efficient Thermally Activat... (0.98 MiB) view on ChemRxiv • download file 


\section{Supporting Information}

Extracting Design Principles for Efficient Thermally Activated Delayed Fluorescence (TADF) from a Simple Four-State Model

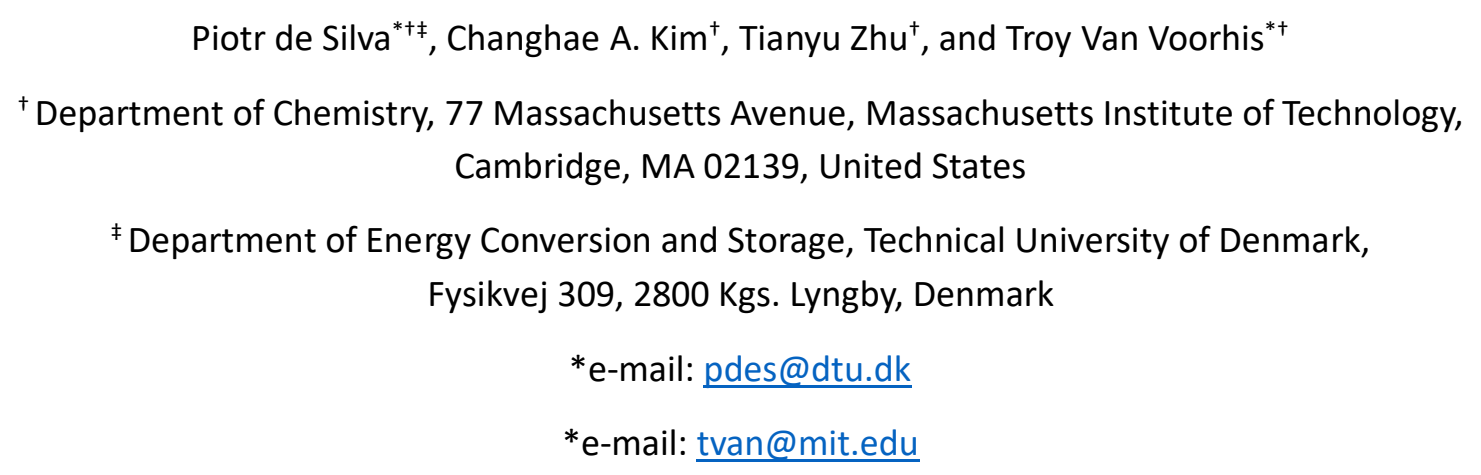

\section{Table of Contents}

Approximations behind the four-state Hamiltonian ...............................................2

Analytic expressions for eigenvalues and eigenvectors of the four-state Hamiltonian.............2

Monte Carlo sampling of the parameter space ...........................................................3

Principal Component Analysis ...........................................................................4

Molecular Dynamics ......................................................................................5

Rate of Thermally Activated Delayed Fluorescence .....................................................9

Time-Dependent Density Functional Theory ...........................................................9

Natural Transition Orbitals ................................................................................. 13

Analysis of the orbital transitions ...................................................................... 14

Polarizable Continuum Model ............................................................................ 16

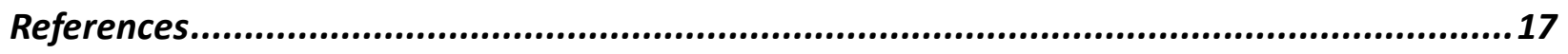




\section{Approximations behind the four-state Hamiltonian}

First, we consider the donor-acceptor system to be isolated; therefore, not interacting with other molecules in the environment. Next, we assume that there are only four excited diabatic states participating in the reverse intersystem crossing and fluorescence. These states can be effectively described by three orbitals localized on either donor or acceptor moieties. The conceptually simplest way to think about these states is to treat them as single electron configurations, which means that they result from some mean-field electronic structure approximation. Due to orthogonalization tails, the orbitals cannot be completely localized on the donor or acceptor. Assuming that such states can still be classified as charge transfer and local excitations, one obtains the four-state Hamiltonian (Eq. 7 in the main text) with coupling elements given by Eqs. 8-11. However, the form of the Hamiltonian in Eq. 7 is not limited to the mean-field approximation, and the diabatic basis states can be treated as correlated many-body states. Such diabatic many-body representation would mean that the states are not necessarily strictly orthogonal and that, in addition to the Hamiltonian, the system' s description requires also an overlap matrix. We assume in the model that the off-diagonal overlaps can be neglected, so the expressions for coupling elements (Eqs. 8-11) still hold. In principle, there might be also some correlation effects that would break the symmetry between $\langle C T 1|\widehat{H}| L E 1\rangle$ and $\langle C T 2|\widehat{H}| L E 2\rangle$, as well as $\langle C T 1|\widehat{H}| L E 2>$ and $<C T 2|\widehat{H}| L E 1>$ coupling elements. In any case, we are assuming that these couplings do not change by flipping the spin of electrons in the excited orbitals.

\section{Analytic expressions for eigenvalues and eigenvectors of the four-state Hamiltonian}

Eigenvalues

$$
\begin{aligned}
& \mathrm{E}_{\mathrm{S}_{1}}=\frac{1}{2}\left(\Delta E+K_{L E}+K_{C T}\right)-\sqrt{\frac{1}{4}\left(\Delta E+K_{L E}+K_{C T}\right)^{2}-\left(\Delta E+K_{L E}\right) K_{C T}+\left(t+K_{X}\right)^{2}} \\
& \mathrm{E}_{\mathrm{S}_{2}}=\frac{1}{2}\left(\Delta E+K_{L E}+K_{C T}\right)+\sqrt{\frac{1}{4}\left(\Delta E+K_{L E}+K_{C T}\right)^{2}-\left(\Delta E+K_{L E}\right) K_{C T}+\left(t+K_{X}\right)^{2}} \\
& \mathrm{E}_{\mathrm{T}_{1}}=\frac{1}{2}\left(\Delta E-K_{L E}-K_{C T}\right)-\sqrt{\frac{1}{4}\left(\Delta E-K_{L E}-K_{C T}\right)^{2}+\left(\Delta E-K_{L E}\right) K_{C T}+\left(t-K_{X}\right)^{2}} \\
& \mathrm{E}_{\mathrm{T}_{2}}=\frac{1}{2}\left(\Delta E-K_{L E}-K_{C T}\right)+\sqrt{\frac{1}{4}\left(\Delta E-K_{L E}-K_{C T}\right)^{2}+\left(\Delta E-K_{L E}\right) K_{C T}+\left(t-K_{X}\right)^{2}}
\end{aligned}
$$


Eigenvectors (unnormalized) in the basis of spin-pure diabatic states

$$
\begin{aligned}
& \left.\left|S_{1}\right\rangle=\left.\left[-\frac{1}{2\left(t+K_{X}\right)}\left(\Delta E-K_{C T}+K_{L E}\right)-\sqrt{\frac{1}{4\left(t+K_{X}\right)^{2}}\left(\Delta E+K_{L E}+K_{C T}\right)^{2}-\frac{\left(\Delta E+K_{L E}\right) K_{C T}}{\left(t+K_{X}\right)^{2}}+1}\right]\right|^{1} C T\right\rangle+\left|{ }^{1} L E\right\rangle \\
& \left|S_{2}\right\rangle=\left[-\frac{1}{2\left(t+K_{X}\right)}\left(\Delta E-K_{C T}+K_{L E}\right)+\sqrt{\frac{1}{4\left(t+K_{X}\right)^{2}}\left(\Delta E+K_{L E}+K_{C T}\right)^{2}-\frac{\left(\Delta E+K_{L E}\right) K_{C T}}{\left(t+K_{X}\right)^{2}}+1}\right]\left|{ }^{1} C T\right\rangle+\left|{ }^{1} L E\right\rangle \\
& \left|T_{1}\right\rangle=\left[-\frac{1}{2\left(t-K_{X}\right)}\left(\Delta E+K_{C T}-K_{L E}\right)-\sqrt{\frac{1}{4\left(t-K_{X}\right)^{2}}\left(\Delta E-K_{L E}-K_{C T}\right)^{2}+\frac{\left(\Delta E-K_{L E}\right) K_{C T}}{\left(t-K_{X}\right)^{2}}+1}\right]\left|{ }^{3} C T\right\rangle+\left|{ }^{3} L E\right\rangle \\
& \left.\left.\left|T_{2}\right\rangle=\left.\left[-\frac{1}{2\left(t-K_{X}\right)}\left(\Delta E+K_{C T}-K_{L E}\right)+\sqrt{\frac{1}{4\left(t-K_{X}\right)^{2}}\left(\Delta E-K_{L E}-K_{C T}\right)^{2}+\frac{\left(\Delta E-K_{L E}\right) K_{C T}}{\left(t-K_{X}\right)^{2}}+1}\right]\right|^{3} C T\right\rangle+\left.\right|^{3} L E\right\rangle
\end{aligned}
$$

\section{Monte Carlo sampling of the parameter space}

\section{Sampling ranges}

To sample the parameter space of the four-state Hamiltonian, we made some arbitrary choices about the relevant ranges of parameters. Typically, the HOMO->LUMO transition would be a CT state and $\Delta E>0$; however, we do not preclude a situation where the energy of the mixed LE is lower than CT and allow for either sign of $\Delta E$. The different character of the CT and LE states is directly reflected in the exchange couplings which we fix to have positive values such that $K_{C T}<K_{L E}$. The distinction between a CT and LE state needs to be somewhat arbitrary, and we choose $K=0.25 \mathrm{eV}$ as the cut-off value for the CT/LE classification. The couplings $t$ and $K_{X}$ can have either sign which is to some extent arbitrary. Changing the phase of $\phi_{L}$ or $\phi_{L+1}$ will flip the sign of both $t$ and $K_{X}$ simultaneously without changing the other parameters, so only the relative sign matters for the model. We expect the average absolute value of $t$ to be larger than $K_{X}$ since it is a sum of a one-electron hopping integral and an exchange-like twoelectron integral, while $K_{X}$ consists of only the latter. Based on these considerations we drew the Hamiltonian parameters from uniform distributions over the following ranges: $\Delta E \in[-2,2] \mathrm{eV} ; K_{C T} \in$ $[0,0.25] \mathrm{eV} ; K_{L E} \in[0.25,2] \mathrm{eV} ; t \in[-2,2] \mathrm{eV} ; K_{X} \in[-1,1] \mathrm{eV}$.

\section{Data filtering}

We have set arbitrary cut-off values to keep only those combinations of parameters that lead to small singlet-triplet gaps, appreciable LE component in the $S_{1}$ state, and significant difference in the electronic character of $S_{1}$ and $T_{1}$. Typically, the reported TADF emitters have singlet-triplet gaps up to $200 \mathrm{meV}$, and computational studies suggest that $S_{1}^{L E}$ and $\triangle C T$ in the order of $10 \%$ may be sufficient for TADF activity ${ }^{1}$. For this study we apply more stringent criteria, namely $\Delta E_{S T}<0.1 \mathrm{eV}, S_{1}^{L E}>0.25$, and $\Delta C T>0.4$, where the latter two values approximately correspond to maxima in Figure $2 \mathrm{~b}$ and Figure $2 \mathrm{c}$. 


\section{Principal Component Analysis}

The Principal Component Analysis (PCA) was performed on a covariance matrix of the parameter vectors in the filtered set. The original parameters of the four-state model were replaced by their combinations which appear directly in the singlet and triplet Hamiltonians, i.e.

$$
\begin{aligned}
& x_{1}=\Delta E+K_{L E} \\
& \mathrm{x}_{2}=\Delta E-K_{L E} \\
& x_{3}=K_{C T} \\
& x_{4}=t+K_{X} \\
& \mathrm{x}_{5}=t-K_{X}
\end{aligned}
$$

The resulting principal components $c_{i}$ had the following coefficient in the original basis (coefficients smaller than 0.005 have been suppressed)

$$
\begin{aligned}
& c_{1}=0.99 x_{4}+0.143 x_{5} \\
& c_{2}=0.959 x_{1}+0.282 x_{2} \\
& c_{3}=0.282 x_{1}-0.959 x_{2}+0.01 x_{5} \\
& c_{4}=0.01 x_{2}-0.143 x_{4}+0.99 x_{5} \\
& c_{5}=x_{3}
\end{aligned}
$$

and the following singular values: $\lambda_{1}=255.29, \lambda_{2}=109.78, \lambda_{3}=55.04, \lambda_{4}=34.60, \lambda_{5}=9.33$, which account for $0.799,0.148,0.037,0.015,0.001$ of the total variance, respectively.

The principal components with the smallest singular values contribute the most to the satisfaction of the filtering criterion, so they represent the combinations of parameters that are prerequisites for TADF. The variability within the filtered set is explained the most by principal components with the largest singular values, so these combinations are responsible for fine-tuning of the TADF efficiency. The principal component with the smallest singular value and negligible contribution to the variance is $K_{C T}$. It reflects the fact that the exchange integral is in general small for CT states and efficient solutions can be found in the entire sampled range. The next component accounts for only $1.5 \%$ of the variance and is dominated by the $t-K_{X}$ combination, which means that along with $K_{C T}$ this parameter determines whether TADF is possible at all. Indeed, the histogram in Figure S1a reveals that the distribution of this parameter is strongly peaked around $0 \mathrm{eV}$ with standard deviation $0.38 \mathrm{eV}$. The next smallest principal component accounts for $3.7 \%$ of the variance and is dominated by the $\Delta E-K_{L E}$ combination. Figure S1b shows that the distribution of this parameter, which has a mean value $-1 \mathrm{eV}$ and standard deviation $0.46 \mathrm{eV}$. It also appears that $K_{L E}$ has to be larger than $\Delta E$ as there are almost no solutions where the opposite is true, and the distribution is noticeably negatively skewed. The remaining two principal components correspond mostly to $\Delta E+K_{L E}$ and $t+K_{X}$ combinations, where the latter accounts for as much as $80 \%$ of the variance, so it is the main source of variation in the filtered data. 

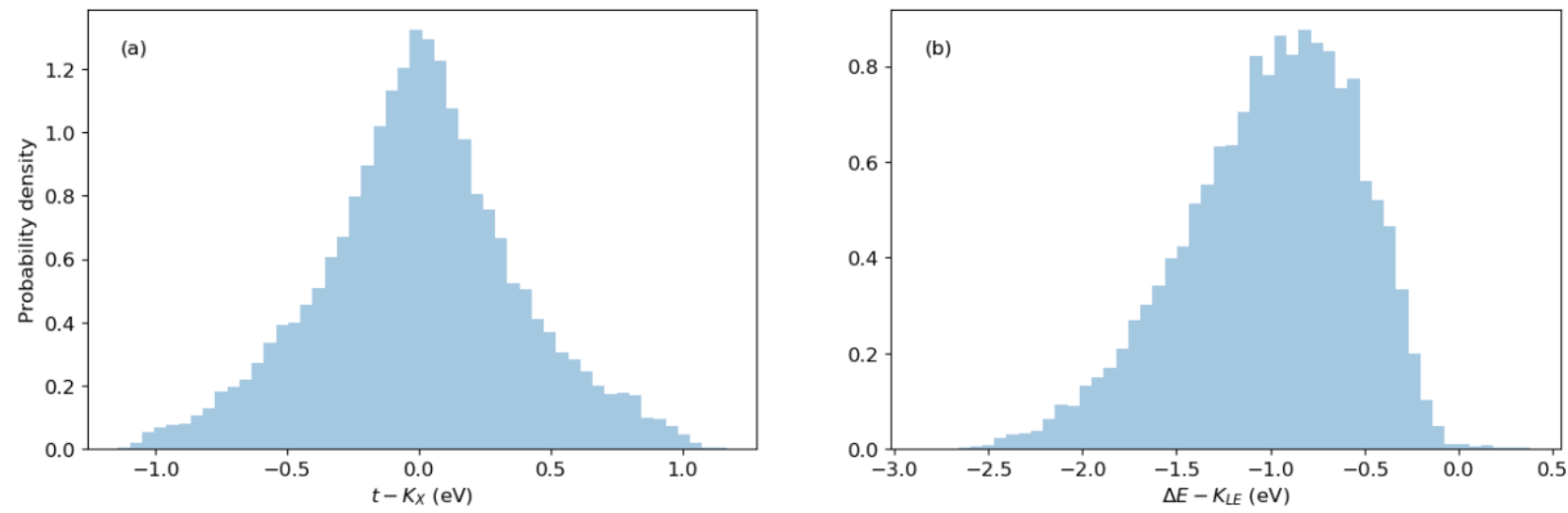

Figure S1 Distributions of $t-K_{X}$ and $\Delta E-K_{L E}$ parameters in the filtered data set.

This analysis of the importance of parameters is somewhat biased by the judicious but arbitrary choice of distribution ranges from which the parameters were sampled. Therefore, the analysis was repeated on normalized original parameters (z-scores): $z_{i}=\frac{x_{i}-\mu_{i}}{\sigma_{i}}$ to remove the initial bias introduced by different variances of original parameters. The resulting principal components are the following (coefficients smaller than 0.005 have been suppressed):

$$
\begin{aligned}
& \tilde{c}_{1}=0.707 t+0.707 K_{X} \\
& \tilde{c}_{2}=0.705 \Delta E+0.704 K_{L E}+0.083 K_{C T} \\
& \tilde{\epsilon}_{3}=-0.041 \Delta E-0.076 K_{L E}+0.996 K_{C T} \\
& \tilde{c}_{4}=-0.708 \Delta E+0.706 K_{L E}+0.024 K_{C T} \\
& c_{5}=0.707 t-0.707 K_{X}
\end{aligned}
$$

with the following singular values $\tilde{\lambda}_{1}=186.334, \tilde{\lambda}_{2}=164.883, \tilde{\lambda}_{3}=132.84, \tilde{\lambda}_{4}=91.023, \tilde{\lambda}_{5}=$ 26.298 , accounting for $0.392,0.307,0.199,0.094,0.008$ of the total variance, respectively.

The compositions of principal components are essentially the same as in the non-standardized case, but the scaling of data changes the explained variance ratios so that $t-K_{X}$ accounts for the least of the variance (0.8\%), followed by $\Delta E-K_{L E}(9.4 \%), K_{C T}$ (19.9\%), $\Delta E+K_{L E}$ (30.7\%), and $t+K_{X} \quad(39.2 \%)$. Therefore, if all the parameters are treated on an equal footing, clearly small values of $t-K_{X}$ and to lesser extent moderately negative $\Delta E-K_{L E}$ are necessary for TADF.

\section{Molecular Dynamics}

Ground state geometry optimization was carried out in the framework of density functional theory (DFT), using the B3LYP exchange-correlation functional ${ }^{2}$ and the $6-31+G^{*}$ basis $\operatorname{set}^{3-7}$ as implemented in the QChem 4.4 software package. ${ }^{8}$ The $\mathrm{CHELPG}^{9}$ charges were computed at the same level of theory. The Cartesian coordinates of the optimized geometry and the CHELPG charges have been provided in Table S1. 


\begin{tabular}{|c|c|c|c|c|c|c|}
\hline Element & $x(\AA)$ & $y(\AA)$ & $z(\AA)$ & CHELPG & Atom Type & Description \\
\hline$N$ & -0.1771724 & 0.1308221 & -0.0536515 & -0.7177610 & opls_641 & 1,3,5-triazine $\mathrm{N}$ \\
\hline C & -0.2907067 & 0.0594104 & -0.0577637 & 0.7412900 & opls_642 & 1,3,5-triazine C \\
\hline $\mathrm{N}$ & -0.2951423 & -0.0747524 & -0.0593212 & -0.7370840 & opls_641 & 1,3,5-triazine $\mathrm{N}$ \\
\hline C & -0.1764402 & -0.1372672 & -0.0562627 & 0.8040150 & opls_642 & 1,3,5-triazine C \\
\hline $\mathrm{N}$ & -0.0581012 & -0.0741559 & -0.0516787 & -0.7356020 & opls_641 & 1,3,5-triazine $\mathrm{N}$ \\
\hline C & -0.0632419 & 0.0599753 & -0.0505072 & 0.7320700 & opls_642 & 1,3,5-triazine C \\
\hline C & 0.0650247 & 0.1344654 & -0.0446425 & -0.0582920 & opls_145B & biphenyl C1 \\
\hline C & 0.0655603 & 0.2748075 & -0.0376595 & -0.1251780 & opls_145 & benzene $\mathrm{C}$ \\
\hline C & 0.1861568 & 0.3446213 & -0.0320655 & -0.1262380 & opls_145 & benzene $\mathrm{C}$ \\
\hline C & 0.3076415 & 0.2753331 & -0.0336096 & -0.0920130 & opls_145 & benzene $\mathrm{C}$ \\
\hline C & 0.1874834 & 0.0655030 & -0.0459690 & -0.0744690 & opls_145 & benzene $\mathrm{C}$ \\
\hline C & 0.3079171 & 0.1356214 & -0.0406489 & -0.1281280 & opls_145 & benzene $\mathrm{C}$ \\
\hline $\mathrm{H}$ & 0.4020596 & 0.0813016 & -0.0421523 & 0.1148450 & opls_146 & benzene $\mathrm{H}$ \\
\hline $\mathrm{H}$ & 0.1868097 & -0.0428006 & -0.0513049 & 0.1075690 & opls_146 & benzene $\mathrm{H}$ \\
\hline $\mathrm{H}$ & 0.1853611 & 0.4531580 & -0.0264337 & 0.1204580 & opls_146 & benzene $\mathrm{H}$ \\
\hline $\mathrm{H}$ & 0.4015845 & 0.3298761 & -0.0293961 & 0.1156810 & opls_146 & benzene $\mathrm{H}$ \\
\hline $\mathrm{H}$ & -0.0290245 & 0.3278108 & -0.0364674 & 0.1357560 & opls_146 & benzene $\mathrm{H}$ \\
\hline C & -0.1759534 & -0.2855269 & -0.0574480 & -0.1276070 & opls_145B & biphenyl C1 \\
\hline C & -0.2965241 & -0.3575297 & -0.0615792 & -0.1110500 & opls_145 & benzene $\mathrm{C}$ \\
\hline C & -0.2962860 & -0.4967235 & -0.0614839 & -0.1966050 & opls_145 & benzene $\mathrm{C}$ \\
\hline C & -0.1748988 & -0.5670871 & -0.0586419 & 0.4028990 & opls_918 & $\mathrm{N}, \mathrm{N}$-dimethylaniline $\mathrm{C}(\mathrm{NH} 2)$ \\
\hline C & -0.0540563 & -0.4958734 & -0.0554877 & -0.2577960 & opls_145 & benzene $\mathrm{C}$ \\
\hline C & -0.0548582 & -0.3566982 & -0.0541884 & -0.1166240 & opls_145 & benzene $\mathrm{C}$ \\
\hline $\mathrm{H}$ & -0.3903387 & -0.3032020 & -0.0635794 & 0.1332380 & opls_146 & benzene $\mathrm{H}$ \\
\hline $\mathrm{H}$ & -0.3900079 & -0.5515554 & -0.0619349 & 0.1355560 & opls_146 & benzene $\mathrm{H}$ \\
\hline $\mathrm{H}$ & 0.0400378 & -0.5500398 & -0.0555464 & 0.1535730 & opls_146 & benzene $\mathrm{H}$ \\
\hline $\mathrm{H}$ & 0.0385533 & -0.3016960 & -0.0519984 & 0.1464590 & opls_146 & benzene $\mathrm{H}$ \\
\hline C & -0.4194147 & 0.1333345 & -0.0601076 & -0.0559090 & opls_145B & biphenyl C1 \\
\hline C & -0.4207058 & 0.2738567 & -0.0619985 & -0.0843540 & opls_145 & benzene $\mathrm{C}$ \\
\hline C & -0.5416996 & 0.3431940 & -0.0637009 & -0.1321960 & opls_145 & benzene C \\
\hline C & -0.6628331 & 0.2732251 & -0.0633679 & -0.0905420 & opls_145 & benzene C \\
\hline C & -0.6623286 & 0.1333685 & -0.0615400 & -0.1288670 & opls_145 & benzene C \\
\hline C & -0.5414982 & 0.0637343 & -0.0600341 & -0.1128430 & opls_145 & benzene C \\
\hline $\mathrm{H}$ & -0.5402709 & -0.0446840 & -0.0586540 & 0.1252090 & opls_146 & benzene $\mathrm{H}$ \\
\hline
\end{tabular}




\begin{tabular}{|c|c|c|c|c|c|c|}
\hline $\mathrm{H}$ & -0.7570711 & 0.3274058 & -0.0645889 & 0.1152140 & opls_146 & benzene $\mathrm{H}$ \\
\hline $\mathrm{H}$ & -0.7561528 & 0.0785045 & -0.0612874 & 0.1197590 & opls_146 & benzene $\mathrm{H}$ \\
\hline $\mathrm{H}$ & -0.3263898 & 0.3273574 & -0.0621695 & 0.1131400 & opls_146 & octicer \\
\hline $\mathrm{H}$ & -0.5415096 & 0.4518743 & -0.0652907 & 0.1187370 & opls_146 & benzene $\mathrm{H}$ \\
\hline $\mathrm{N}$ & -0.1743323 & -0.7087744 & -0.0587923 & -0.3621580 & opls_587 & indole N1 \\
\hline C & -0.1100683 & -0.7906475 & 0.0352199 & 0.2739950 & opls_594 & indole C8 \\
\hline C & -0.1339998 & -0.9264327 & 0.0018879 & -0.0149390 & opls_595 & indole C9 \\
\hline C & -0.2158921 & -0.9266986 & -0.1176433 & -0.0119650 & opls_595 & indole C9 \\
\hline C & -0.3120495 & -0.7559178 & -0.2661347 & -0.2717900 & opls_593 & indole C7 \\
\hline C & -0.3638388 & -0.8591149 & -0.3444576 & -0.0788970 & opls_592 & dole C6 \\
\hline C & -0.3431816 & -0.9941730 & -0.3108205 & -0.2075590 & opls_591 & indole C5 \\
\hline C & -0.2689765 & -1.0284647 & -0.1979432 & -0.1527430 & opls_590 & indole C4 \\
\hline C & -0.0813888 & -1.0278192 & 0.0829722 & -0.1582400 & opls_590 & dole C4 \\
\hline C & -0.0068041 & -0.9930365 & 0.1954527 & -0.1963340 & opls_591 & indole C5 \\
\hline C & 0.0147864 & -0.8578391 & 0.2278818 & -0.0957790 & opls_592 & le C6 \\
\hline C & -0.0365160 & -0.7550062 & 0.1487574 & -0.2900690 & opls_593 & le C7 \\
\hline C & -0.2390061 & -0.7910665 & -0.1521262 & 0.2533390 & opls_594 & indole C8 \\
\hline $\mathrm{H}$ & -0.0989574 & -1.1323350 & 0.0587324 & 0.1423740 & opls_599 & indole H4 \\
\hline $\mathrm{H}$ & 0.0346372 & -1.0707802 & 0.2590432 & 0.1355690 & opls_600 & indole H5 \\
\hline $\mathrm{H}$ & 0.0724471 & -0.8325453 & 0.3164712 & 0.1257360 & opls_601 & indole H6 \\
\hline $\mathrm{H}$ & 9 & -0.6510878 & 0.1753063 & 0.1 & 02 & H7 \\
\hline $\mathrm{H}$ & -0.3276138 & -0.6521202 & -0.2937596 & 0.1424250 & opls_602 & indole H7 \\
\hline $\mathrm{H}$ & -0.4210393 & -0.8342520 & -0.4334683 & 0.1193510 & opls_601 & indole H6 \\
\hline $\mathrm{H}$ & -0.3849812 & -1.0721943 & -0.3738366 & 0.1356040 & opls_600 & indole H5 \\
\hline $\mathrm{H}$ & -0.2520441 & -1.1328719 & -0.1728020 & 0.1420160 & opls_599 & indole H4 \\
\hline $\mathrm{H}$ & -0.3629621 & 0.0623256 & 0.2920560 & 0.1482280 & opls_146 & benzene $\mathrm{H}$ \\
\hline $\mathrm{H}$ & -0.4094327 & 0.3049320 & 0.2826535 & 0.1569120 & opls_146 & benzene $\mathrm{H}$ \\
\hline $\mathrm{H}$ & -0.1951850 & -0.2894448 & 0.3104549 & 0.1619210 & opls_146 & benzene $\mathrm{H}$ \\
\hline $\mathrm{H}$ & -0.3824010 & -0.4515855 & 0.3092251 & 0.1226910 & opls_146 & benzene $\mathrm{H}$ \\
\hline $\mathrm{H}$ & 0.0251872 & 0.0312985 & 0.3065705 & 0.1697260 & opls_146 & benzene $\mathrm{H}$ \\
\hline $\mathrm{H}$ & 0.2586801 & -0.0490898 & 0.3186254 & 0.1404710 & opls_146 & benzene $\mathrm{H}$ \\
\hline C & -0.3074025 & 0.1300405 & 0.3561811 & -0.2318230 & opls_145 & e C \\
\hline C & -0.2835773 & -0.2701037 & 0.3704517 & -0.2903400 & opls_145 & benze \\
\hline C & -0.3340177 & 0.2668022 & 0.3507469 & -0.1453770 & opls_145 & benzene $\mathrm{C}$ \\
\hline C & -0.3887857 & -0.3615989 & 0.3695699 & -0.0740760 & opls_145 & benzene $\mathrm{C}$ \\
\hline
\end{tabular}




$\begin{array}{lrlllll}\text { C } & 0.0506771 & -0.0492222 & 0.3747772 & -0.3003650 & \text { opls_145 } & \text { benzene C } \\ \text { C } & 0.1824429 & -0.0943736 & 0.3812176 & -0.0952270 & \text { opls_145 } & \text { benzene C } \\ \text { C } & -0.2611513 & 0.3542177 & 0.4314462 & 0.0789740 & \text { opls_263 } & \text { chlorobenzene C(Cl) } \\ \text { C } & 0.2150726 & -0.1994313 & 0.4670469 & 0.0526910 & \text { opls_263 } & \text { chlorobenzene C(Cl) } \\ \text { C } & -0.5032591 & -0.3354795 & 0.4449634 & 0.0606780 & \text { opls_263 } & \text { chlorobenzene C(Cl) } \\ \text { C } & -0.2094671 & 0.0792910 & 0.4431227 & 0.3887900 & \text { opls_918 } & \text { N,N-dimethylaniline C(NH2) } \\ \text { C } & -0.2908085 & -0.1530699 & 0.4477933 & 0.4007680 & \text { opls_918 } & \text { N,N-dimethylaniline C(NH2) } \\ \text { C } & -0.0490429 & -0.1070993 & 0.4549987 & 0.4285840 & \text { opls_918 } & \text { N,N-dimethylaniline C(NH2) } \\ \text { N } & -0.1831153 & -0.0602986 & 0.4489654 & -0.4642580 & \text { opls_902 } & \text { tertiary amine N } \\ \text { Cl } & -0.2933720 & 0.5270910 & 0.4239583 & -0.1396110 & \text { opls_264 } & \text { chlorobenzene Cl } \\ \text { Cl } & 0.3810083 & -0.2575846 & 0.4745096 & -0.1365380 & \text { opls_264 } & \text { chlorobenzene Cl } \\ \text { Cl } & -0.6369042 & -0.4499010 & 0.4429752 & -0.1369440 & \text { opls_264 } & \text { chlorobenzene Cl } \\ \text { C } & 0.1173806 & -0.2589631 & 0.5469530 & -0.0757120 & \text { opls_145 } & \text { benzene C } \\ \text { C } & -0.1627541 & 0.3060616 & 0.5178761 & -0.1060680 & \text { opls_145 } & \text { benzene C } \\ \text { C } & -0.5128685 & -0.2194098 & 0.5218289 & -0.1019440 & \text { opls_145 } & \text { benzene C } \\ \text { C } & -0.0138597 & -0.2121586 & 0.5413605 & -0.3212080 & \text { opls_145 } & \text { benzene C } \\ \text { C } & -0.4065416 & -0.1292215 & 0.5237448 & -0.2987640 & \text { opls_145 } & \text { benzene C } \\ \text { C } & -0.1378692 & 0.1689937 & 0.5241048 & -0.2932570 & \text { opls_145 } & \text { benzene C } \\ \text { H } & 0.1436894 & -0.3401015 & 0.6140856 & 0.1336590 & \text { opls_146 } & \text { benzene H } \\ \text { H } & -0.6019486 & -0.2002259 & 0.5808083 & 0.1401700 & \text { opls_146 } & \text { benzene H } \\ \text { H } & -0.1069738 & 0.3746853 & 0.5808106 & 0.1393450 & \text { opls_146 } & \text { benzene H } \\ \text { H } & -0.0897067 & -0.2575724 & 0.6043950 & 0.1609010 & \text { opls_146 } & \text { benzene H } \\ \text { H } & -0.4134755 & -0.0394764 & 0.5844489 & 0.1550840 & \text { opls_146 } & \text { benzene H } \\ \text { H } & -0.0621588 & 0.1312941 & 0.5921804 & 0.1557080 & \text { opls_146 } & \text { benzene H }\end{array}$

Table S1 Cartesian coordinates of DFT optimized geometry, the CHELPG charges, and the atom type assignments.

The molecular dynamics (MD) simulations were carried out using the OPLS-aa force field ${ }^{10}$ as implemented in the GROMACS 5.1.2 software package. ${ }^{11}$ Since not every atom could be assigned to the exact atom type, assignments were made to best describe the chemical environment of each atom. The atom type assignments can be found in Table S1. The equilibrium bond lengths, bond angles, and dihedral angles were parametrized to the DFT optimized geometry, and the default charges were replaced with the CHELPG charges. Periodic boundary conditions were enforced so that the complex was $1.0 \mathrm{~nm}$ from the walls of the cubic box at the beginning of the simulation. Although an NVT ensemble was used, a separate equilibration stage was deemed unnecessary, since the focus was not on the thermodynamics. The NoseHoover thermostat ${ }^{12-14}$ was set at $300 \mathrm{~K}$ during regular MD runs and at $400 \mathrm{~K}$ during runs with modified Lennard-Jones parameters. 
Since the initial results of TD-DFT suggested that the oscillator strength, rather than $\Delta \mathrm{E}_{S T}$, was the limiting factor of the TADF rate, many of the MD simulations had the Lennard-Jones parameters of the OPLS-aa force field modified to push the donor and the acceptor moieties closer together and increase the HOMOLUMO overlap. The Lennard-Jones potential has the form:

$$
V_{\mathrm{LJ}}(r)=4 \varepsilon\left[\left(\frac{\sigma}{r}\right)^{12}-\left(\frac{\sigma}{r}\right)^{6}\right]
$$

where $\varepsilon$ and $\sigma$ are atom-specific parameters that control the depth and the position of the potential well, respectively. Keeping the $\varepsilon$-parameter of each atom at 1.5 times the default value, the $\sigma$-parameters were scaled down by a number of different factors.

Unless otherwise mentioned, snapshots were taken at rather short intervals of $0.1 \mathrm{ps}$, but they were sampled at longer intervals for TD-DFT calculations. After the initial sampling, the temporal correlation between snapshots was used to select the intervals on which to focus the search. Whenever a simulation exhibited a transition between a large oscillator strength and a small $\Delta \mathrm{E}_{\mathrm{ST}}$ or an extended period of either, the more frames in that part of the simulation were submitted to TD-DFT, with the hope that the trajectory would encounter a conformation of both a large oscillator strength and a small $\Delta \mathrm{E}_{\mathrm{ST}}$.

\section{Rate of Thermally Activated Delayed Fluorescence}

Eq. (20) of the main article assumes that the excited state populations have thermalized and that only the $S_{1}$ and the $T_{1}$ states are populated to a significant extent. On the other hand, if the $T_{2}$ state were lower in energy than the $S_{1}$ state, then the $T_{2}$ state should be included in the denominator. ${ }^{15}$ The formula becomes

$$
\begin{aligned}
k_{\mathrm{TADF}} & =\frac{k_{\mathrm{F}}\left[S_{1}\right]}{\left[S_{1}\right]+\left[T_{1}\right]}=\frac{k_{\mathrm{F}}}{1+K_{1}+K_{2}} \\
K_{1} & \equiv \frac{\left[T_{1}\right]}{\left[S_{1}\right]}=3 \exp \left(\frac{\Delta E_{S T_{1}}}{k_{\mathrm{B}} T}\right) \\
K_{2} & \equiv \frac{\left[T_{2}\right]}{\left[S_{1}\right]}=3 \exp \left(\frac{\Delta E_{S T_{2}}}{k_{\mathrm{B}} T}\right)
\end{aligned}
$$

However, the correction was neglected in the main article, because taking the $T_{2}$ state into consideration neither entailed an order-of-magnitude correction to a TADF rate nor altered the statistics in a meaningful way. Examples can be found in the following section.

\section{Time-Dependent Density Functional Theory}

The excited states in the selected conformations were computed in the framework of time-dependent density functional theory (TD-DFT), using the same level of theory as geometry optimization. In the first MD simulation, which used the default Lennard-Jones parameters, some twenty conformations were sampled. The TD-DFT results and the estimated rates have been provided in Table S2. The TADF rate could already be seen undergoing order-of-magnitude fluctuations. The shortest TADF lifetime to be obtained at 
this point was $13 \mu \mathrm{s}$ (oscillator strength of $1.1 \times 10-3$ and $\Delta \mathrm{E}_{S T}$ of $0.01 \mathrm{eV}$ ), to be contrasted with the DFT optimized geometry, which was estimated to have a TADF lifetime of $1.6 \mathrm{~ms}$ (oscillator strength of 1.8x105 and $\Delta \mathrm{E}_{S T}$ of $0.02 \mathrm{eV}$ ). Since $\Delta \mathrm{E}_{S T}<\mathrm{k}_{\mathrm{B}} \mathrm{T}=25.7 \mathrm{meV}$ was not difficult to obtain in the model system, it was decided that the oscillator strength, rather than $\triangle \mathrm{E}_{S T}$, was the limiting factor of the TADF rate.

\begin{tabular}{|c|c|c|c|c|c|c|c|c|}
\hline Frame & $\mathrm{T}_{1}(\mathrm{eV})$ & $\mathrm{T}_{2}(\mathrm{eV})$ & $\mathrm{T}_{\mathrm{n}}<\mathrm{S}_{1}$ & $\mathrm{~S}_{1}(\mathrm{eV})$ & $f_{10}$ & $\mathrm{PF}^{\mathrm{a}}(\mu \mathrm{s})$ & $\operatorname{TADF}^{\mathrm{b}}(\mu \mathrm{s})$ & $\operatorname{TADF}^{\mathrm{c}}(\mu \mathrm{s})$ \\
\hline $\mathrm{opt}^{\mathrm{d}}$ & 2.64 & 2.66 & 2 & 2.66 & $1.1 \times 10^{-3}$ & 2.8 & $1.3 \times 10^{1}$ & $1.4 \times 10^{1}$ \\
\hline 401 & 2.77 & 2.83 & 1 & 2.78 & $1.8 \times 10^{-3}$ & 1.7 & $1.5 \times 10^{1}$ & $1.6 \times 10^{1}$ \\
\hline 0 & 2.68 & 2.74 & 1 & 2.70 & $7.9 \times 10^{-4}$ & 3.7 & $2.5 \times 10^{1}$ & $2.6 \times 10^{1}$ \\
\hline 800 & 2.80 & 2.89 & 1 & 2.81 & $5.5 \times 10^{-4}$ & 6.1 & $3.1 \times 10^{1}$ & $4.4 \times 10^{1}$ \\
\hline 201 & 2.61 & 2.63 & 1 & 2.62 & $4.8 \times 10^{-4}$ & 6.0 & $3.3 \times 10^{1}$ & $3.4 \times 10^{1}$ \\
\hline 300 & 2.84 & 2.93 & 1 & 2.85 & $6.4 \times 10^{-4}$ & 4.2 & $4.0 \times 10^{1}$ & $5.3 \times 10^{1}$ \\
\hline 400 & 2.90 & 2.92 & 1 & 2.92 & $6.1 \times 10^{-4}$ & 4.6 & $4.9 \times 10^{1}$ & $6.0 \times 10^{1}$ \\
\hline 200 & 2.82 & 2.86 & 1 & 2.85 & $7.7 \times 10^{-4}$ & 3.3 & $6.7 \times 10^{1}$ & $1.2 \times 10^{2}$ \\
\hline 202 & 2.94 & 2.95 & 2 & 2.99 & $3.1 \times 10^{-4}$ & 8.9 & $8.2 \times 10^{1}$ & $1.0 \times 10^{2}$ \\
\hline 901 & 2.87 & 2.91 & 1 & 2.90 & $9.5 \times 10^{-5}$ & $2.6 \times 10^{1}$ & $1.3 \times 10^{2}$ & $2.1 \times 10^{2}$ \\
\hline 2 & 3.05 & 3.05 & 1 & 3.05 & $4.0 \times 10^{-4}$ & 6.7 & $2.9 \times 10^{3}$ & $2.9 \times 10^{3}$ \\
\hline 601 & 2.81 & 2.90 & 3 & 2.94 & $5.2 \times 10^{-4}$ & 4.9 & $3.2 \times 10^{3}$ & $3.2 \times 10^{3}$ \\
\hline 701 & 2.88 & 3.00 & 3 & 3.02 & $5.1 \times 10^{-4}$ & 4.7 & $5.0 \times 10^{3}$ & $5.1 \times 10^{3}$ \\
\hline 301 & 2.92 & 3.02 & 4 & 3.07 & $3.9 \times 10^{-3}$ & $6.0 \times 10^{-1}$ & $9.4 \times 10^{3}$ & $1.4 \times 10^{4}$ \\
\hline 100 & 2.93 & 2.95 & 4 & 3.15 & $1.3 \times 10^{-3}$ & 1.9 & $1.9 \times 10^{4}$ & $1.9 \times 10^{4}$ \\
\hline 500 & 2.87 & 3.03 & 3 & 3.08 & $8.2 \times 10^{-5}$ & $3.2 \times 10^{1}$ & $2.9 \times 10^{4}$ & $3.0 \times 10^{4}$ \\
\hline 101 & 2.81 & 2.92 & 3 & 2.96 & $1.1 \times 10^{-2}$ & $1.9 \times 10^{-1}$ & $5.6 \times 10^{4}$ & $5.6 \times 10^{4}$ \\
\hline 102 & 2.69 & 2.97 & 2 & 2.99 & $6.4 \times 10^{-4}$ & 3.8 & $1.1 \times 10^{5}$ & $1.2 \times 10^{5}$ \\
\hline 501 & 2.87 & 2.92 & 5 & 3.10 & $2.7 \times 10^{-3}$ & 1.0 & $4.0 \times 10^{5}$ & $4.0 \times 10^{5}$ \\
\hline 600 & 2.61 & 2.89 & 3 & 2.92 & $1.6 \times 10^{-4}$ & $1.6 \times 10^{1}$ & $9.7 \times 10^{5}$ & $9.7 \times 10^{5}$ \\
\hline 1 & 2.72 & 2.96 & 2 & 2.97 & $4.8 \times 10^{-5}$ & $4.6 \times 10^{1}$ & $1.0 \times 10^{6}$ & $1.5 \times 10^{6}$ \\
\hline 700 & 3.00 & 3.01 & 5 & 3.23 & $1.7 \times 10^{-4}$ & $1.5 \times 10^{1}$ & $1.7 \times 10^{6}$ & $1.7 \times 10^{6}$ \\
\hline 801 & 2.76 & 2.95 & 3 & 3.03 & $4.4 \times 10^{-4}$ & 5.0 & $6.6 \times 10^{7}$ & $7.4 \times 10^{7}$ \\
\hline 900 & 2.86 & 2.91 & 4 & 3.25 & $1.8 \times 10^{-5}$ & $1.8 \times 10^{2}$ & $1.6 \times 10^{3}$ & $2.2 \times 10^{3}$ \\
\hline \multicolumn{9}{|c|}{ a The prompt fluorescence lifetime according to Equation 19 of the main article. } \\
\hline \multicolumn{9}{|c|}{${ }^{b}$ The TADF lifetime according to Equation 20 of the main article. } \\
\hline \multicolumn{9}{|c|}{${ }^{\mathrm{C}}$ The TADF lifetime according to Equation S1. } \\
\hline
\end{tabular}

Table S2 TD-DFT results on the MD simulation with the default Lennard-Jones parameters. Here, the snapshots were taken at 1ps intervals. Except for the DFT-optimized geometry, the conformations have been sorted in the order of increasing TADF lifetime. 
In the MD simulations with modified Lennard-Jones parameters, the $\varepsilon$-parameter of each atom was kept at 1.5 times the default value, while the $\sigma$-parameter was scaled down by a number of different values (see Molecular Dynamics). Over a thousand conformations have been sampled. As such, only the conformations with the fastest TADF in each simulation have been listed in Table S3, Table S4, Table S5, Table S6, and Table S7.

\begin{tabular}{rrrrrllll} 
Frame & $\mathrm{T}_{1}(\mathrm{eV})$ & $\mathrm{T}_{2}(\mathrm{eV})$ & $\mathrm{T}_{\mathrm{n}}<\mathrm{S}_{1}$ & $\mathrm{~S}_{1}(\mathrm{eV})$ & \multicolumn{1}{c}{$f_{10}$} & \multicolumn{1}{c}{$\mathrm{PF}(\mu \mathrm{s})$} & \multicolumn{1}{c}{ TADF $(\mu \mathrm{s})$} & TADF $(\mu \mathrm{s})$ \\
0 & 2.49 & 2.55 & 1 & 2.53 & $8.4 \times 10^{-3}$ & $4.3 \times 10^{-1}$ & 6.1 & 6.6 \\
20 & 2.53 & 2.54 & 1 & 2.53 & $1.7 \times 10^{-3}$ & 2.2 & $1.0 \times 10^{1}$ & $1.5 \times 10^{1}$ \\
8 & 2.58 & 2.66 & 1 & 2.60 & $2.7 \times 10^{-3}$ & 1.3 & $1.2 \times 10^{1}$ & $1.2 \times 10^{1}$ \\
160 & 2.54 & 2.67 & 1 & 2.55 & $1.2 \times 10^{-3}$ & 3.0 & $1.6 \times 10^{1}$ & $1.6 \times 10^{1}$ \\
12 & 2.57 & 2.65 & 1 & 2.58 & $1.4 \times 10^{-3}$ & 3.0 & $1.7 \times 10^{1}$ & $1.7 \times 10^{1}$ \\
2 & 2.43 & 2.47 & 2 & 2.49 & $4.6 \times 10^{-3}$ & $8.1 \times 10^{-1}$ & $2.4 \times 10^{1}$ & $3.0 \times 10^{1}$ \\
4 & 2.19 & 2.28 & 1 & 2.21 & $8.6 \times 10^{-4}$ & 5.5 & $3.6 \times 10^{1}$ & $3.6 \times 10^{1}$ \\
110 & 2.17 & 2.57 & 1 & 2.17 & $4.8 \times 10^{-4}$ & $1.0 \times 10^{1}$ & $4.4 \times 10^{1}$ & $4.4 \times 10^{1}$
\end{tabular}

Table S3 TD-DFT results on the MD simulation with modified Lennard-Jones parameters. The $\sigma$ parameter of each atom was set to 0.9 times the default value.

\begin{tabular}{rrrrrllll} 
Frame & $\mathrm{T}_{1}(\mathrm{eV})$ & $\mathrm{T}_{2}(\mathrm{eV})$ & $\mathrm{T}_{\mathrm{n}}<\mathrm{S}_{1}$ & $\mathrm{~S}_{1}(\mathrm{eV})$ & \multicolumn{1}{c}{$f_{10}$} & \multicolumn{1}{c}{$\mathrm{PF}(\mu \mathrm{s})$} & \multicolumn{1}{c}{ TADF $(\mu \mathrm{s})$} & \multicolumn{1}{c}{ TADF $(\mu \mathrm{s})$} \\
\hline 51 & 2.38 & 2.75 & 1 & 2.41 & $9.9 \times 10^{-3}$ & $4.0 \times 10^{-1}$ & 3.6 & 3.6 \\
81 & 2.60 & 2.67 & 1 & 2.63 & $7.3 \times 10^{-3}$ & $4.6 \times 10^{-1}$ & 5.0 & 5.3 \\
90 & 2.11 & 2.52 & 1 & 2.12 & $3.6 \times 10^{-3}$ & 1.4 & $1.0 \times 10^{1}$ & $1.0 \times 10^{1}$ \\
150 & 2.52 & 2.59 & 1 & 2.57 & $5.00 \times 10^{-3}$ & $7.0 \times 10^{-1}$ & $1.3 \times 10^{1}$ & $1.4 \times 10^{1}$ \\
53 & 2.65 & 2.68 & 2 & 2.69 & $3.4 \times 10^{-3}$ & $9.3 \times 10^{-1}$ & $1.4 \times 10^{1}$ & $1.8 \times 10^{1}$ \\
92 & 2.68 & 2.74 & 2 & 2.77 & $2.2 \times 10^{-2}$ & $1.4 \times 10^{-1}$ & $1.4 \times 10^{1}$ & $1.5 \times 10^{1}$ \\
65 & 2.57 & 2.82 & 1 & 2.66 & $2.2 \times 10^{-2}$ & $1.5 \times 10^{-1}$ & $1.4 \times 10^{1}$ & $1.4 \times 10^{1}$ \\
88 & 2.27 & 2.45 & 1 & 2.28 & $1.6 \times 10^{-3}$ & 2.8 & $1.6 \times 10^{1}$ & $1.6 \times 10^{1}$
\end{tabular}

Table S4 TD-DFT results on the MD simulation with modified Lennard-Jones parameters. The $\sigma$ parameter of each atom was set to 0.8 times the default value.

\begin{tabular}{rrrrrllll} 
Frame & $\mathrm{T}_{1}(\mathrm{eV})$ & $\mathrm{T}_{2}(\mathrm{eV})$ & $\mathrm{T}_{\mathrm{n}}<\mathrm{S}_{1}$ & $\mathrm{~S}_{1}(\mathrm{eV})$ & \multicolumn{1}{c}{$f_{10}$} & \multicolumn{1}{c}{$\mathrm{PF}(\mu \mathrm{s})$} & \multicolumn{1}{c}{ TADF $(\mu \mathrm{s})$} & TADF $(\mu \mathrm{s})$ \\
212 & 2.38 & 2.40 & 1 & 2.40 & $7.4 \times 10^{-3}$ & $5.4 \times 10^{-1}$ & 3.7 & 5.2 \\
165 & 2.47 & 2.73 & 1 & 2.48 & $3.7 \times 10^{-3}$ & 1.0 & 5.0 & 5.0 \\
191 & 2.57 & 2.63 & 1 & 2.58 & $4.0 \times 10^{-3}$ & $8.7 \times 10^{-1}$ & 5.4 & 5.8 \\
10 & 2.31 & 2.42 & 1 & 2.36 & $1.2 \times 10^{-2}$ & $3.5 \times 10^{-1}$ & 8.2 & 8.3 \\
213 & 2.67 & 2.69 & 2 & 2.74 & $1.7 \times 10^{-2}$ & $1.8 \times 10^{-1}$ & 8.2 & $1.2 \times 10^{1}$ \\
214 & 2.32 & 2.52 & 1 & 2.42 & $4.1 \times 10^{-2}$ & $9.7 \times 10^{-2}$ & 9.2 & 9.2
\end{tabular}




$\begin{array}{lllllllll}163 & 2.66 & 2.72 & 1 & 2.67 & 2.1 \times 10^{-3} & 1.6 & 9.9 & 1.1 \times 10^{1} \\ 190 & 2.44 & 2.60 & 1 & 2.46 & 2.5 \times 10^{-3} & 1.6 & 1.0 \times 10^{1} & 1.0 \times 10^{1}\end{array}$

Table S5 TD-DFT results on the MD simulation with modified Lennard-Jones parameters. The $\sigma$ parameter of each atom was set to 0.75 times the default value.

\begin{tabular}{ccccccccc} 
Frame & $\mathrm{T}_{1}(\mathrm{eV})$ & $\mathrm{T}_{2}(\mathrm{eV})$ & $\mathrm{T}_{\mathrm{n}}<\mathrm{S}_{1}$ & $\mathrm{~S}_{1}(\mathrm{eV})$ & $f_{10}$ & $\mathrm{PF}(\mu \mathrm{s})$ & TADF $(\mu \mathrm{s})$ & TADF $(\mu \mathrm{s})$ \\
438 & 2.41 & 2.43 & 2 & 2.43 & $9.6 \times 10^{-3}$ & $4.1 \times 10^{-1}$ & 2.7 & 4.1 \\
363 & 2.07 & 2.44 & 1 & 2.10 & $1.8 \times 10^{-2}$ & $2.9 \times 10^{-1}$ & 2.8 & 2.8 \\
368 & 1.96 & 2.22 & 1 & 2.00 & $2.6 \times 10^{-2}$ & $2.2 \times 10^{-1}$ & 3.1 & 3.1 \\
366 & 2.23 & 2.59 & 1 & 2.24 & $9.0 \times 10^{-3}$ & $5.1 \times 10^{-1}$ & 3.4 & 3.4 \\
173 & 2.36 & 2.42 & 1 & 2.39 & $1.2 \times 10^{-2}$ & $3.5 \times 10^{-1}$ & 3.5 & 3.8 \\
361 & 2.16 & 2.38 & 1 & 2.22 & $4.1 \times 10^{-2}$ & $1.1 \times 10^{-1}$ & 3.6 & 3.6 \\
185 & 2.19 & 2.42 & 1 & 2.24 & $1.7 \times 10^{-2}$ & $2.8 \times 10^{-1}$ & 5.2 & 5.2 \\
171 & 2.56 & 2.60 & 1 & 2.59 & $6.2 \times 10^{-3}$ & $5.5 \times 10^{-1}$ & 5.5 & 6.6 \\
\hline 749 & 2.46 & 2.53 & 1 & 2.48 & $1.4 \times 10^{-2}$ & $2.8 \times 10^{-1}$ & 2.0 & 2.1 \\
800 & 2.48 & 2.51 & 1 & 2.51 & $2.4 \times 10^{-2}$ & $1.5 \times 10^{-1}$ & 2.1 & 2.6 \\
819 & 2.21 & 2.47 & 1 & 2.25 & $1.9 \times 10^{-2}$ & $2.4 \times 10^{-1}$ & 3.2 & 3.2 \\
657 & 2.21 & 2.37 & 1 & 2.22 & $1.0 \times 10^{-2}$ & $4.6 \times 10^{-1}$ & 3.3 & 3.3 \\
315 & 2.35 & 2.41 & 1 & 2.40 & $2.1 \times 10^{-2}$ & $1.9 \times 10^{-1}$ & 3.7 & 4.1 \\
666 & 2.30 & 2.55 & 1 & 2.32 & $9.5 \times 10^{-3}$ & $4.5 \times 10^{-1}$ & 3.8 & 3.8 \\
219 & 2.40 & 2.48 & 1 & 2.42 & $5.1 \times 10^{-3}$ & $7.8 \times 10^{-1}$ & 4.4 & 4.5 \\
801 & 2.25 & 2.42 & 1 & 2.30 & $2.1 \times 10^{-2}$ & $2.1 \times 10^{-1}$ & 4.4 & 4.4
\end{tabular}

Table S6 TD-DFT results on the MD simulation with modified Lennard-Jones parameters. The $\sigma$ parameter of each atom was set to 0.7 times the default value.

\begin{tabular}{rrrrrllll} 
Frame & $\mathrm{T}_{1}(\mathrm{eV})$ & $\mathrm{T}_{2}(\mathrm{eV})$ & $\mathrm{T}_{\mathrm{n}}<\mathrm{S}_{1}$ & $\mathrm{~S}_{1}(\mathrm{eV})$ & \multicolumn{1}{c}{$f_{10}$} & \multicolumn{1}{c}{$\mathrm{PF}(\mu \mathrm{s})$} & \multicolumn{1}{c}{ TADF $(\mu \mathrm{s})$} & TADF $(\mu \mathrm{s})$ \\
155 & 1.94 & 2.20 & 1 & 2.04 & $2.0 \times 10^{-2}$ & $2.8 \times 10^{-1}$ & $4.2 \times 10^{1}$ & $4.2 \times 10^{1}$ \\
115 & 2.02 & 2.33 & 1 & 2.06 & $1.1 \times 10^{-3}$ & 4.8 & $8.4 \times 10^{1}$ & $8.4 \times 10^{1}$ \\
195 & 1.92 & 2.10 & 1 & 2.06 & $1.4 \times 10^{-2}$ & $3.9 \times 10^{-1}$ & $3.5 \times 10^{2}$ & $3.5 \times 10^{2}$ \\
125 & 2.12 & 2.17 & 3 & 2.28 & $1.9 \times 10^{-2}$ & $2.3 \times 10^{-1}$ & $3.9 \times 10^{2}$ & $4.4 \times 10^{2}$ \\
455 & 1.51 & 1.65 & 1 & 1.58 & $1.0 \times 10^{-3}$ & 9.2 & $4.1 \times 10^{2}$ & $4.1 \times 10^{2}$ \\
305 & 1.55 & 1.85 & 1 & 1.71 & $2.9 \times 10^{-2}$ & $2.7 \times 10^{-1}$ & $5.0 \times 10^{2}$ & $5.0 \times 10^{2}$ \\
185 & 1.15 & 1.18 & 2 & 1.23 & $1.7 \times 10^{-3}$ & 8.7 & $7.8 \times 10^{2}$ & $9.6 \times 10^{2}$ \\
6 & 1.95 & 2.11 & 2 & 2.12 & $1.3 \times 10^{-2}$ & $3.8 \times 10^{-1}$ & $7.9 \times 10^{2}$ & $7.9 \times 10^{2}$
\end{tabular}

Table S7 TD-DFT results on the MD simulation with modified Lennard-Jones parameters. The $\sigma$ parameter of each atom was set to 0.6 times the default value. 


\section{Natural Transition Orbitals}

The conformers in Figure 6 of the main article correspond to frames 438 and 819 in Table S6. Here, two more representative conformations are examined. With the ISO value fixed at 0.02 , the dominant NTO pairs in the $S_{1}$ and the $T_{1}$ excited states have been visualized in Figure S2 and Figure S3.

Figure S2 corresponds to frame 366 in Table S6. A large fraction of the hole orbital is delocalized onto the acceptor molecule, showing that the $S_{1}$ state of the donor-acceptor complex can have a significant LE contribution. The differences between the $T_{1}$ and the $S_{1}$ states are less obvious in this conformation.
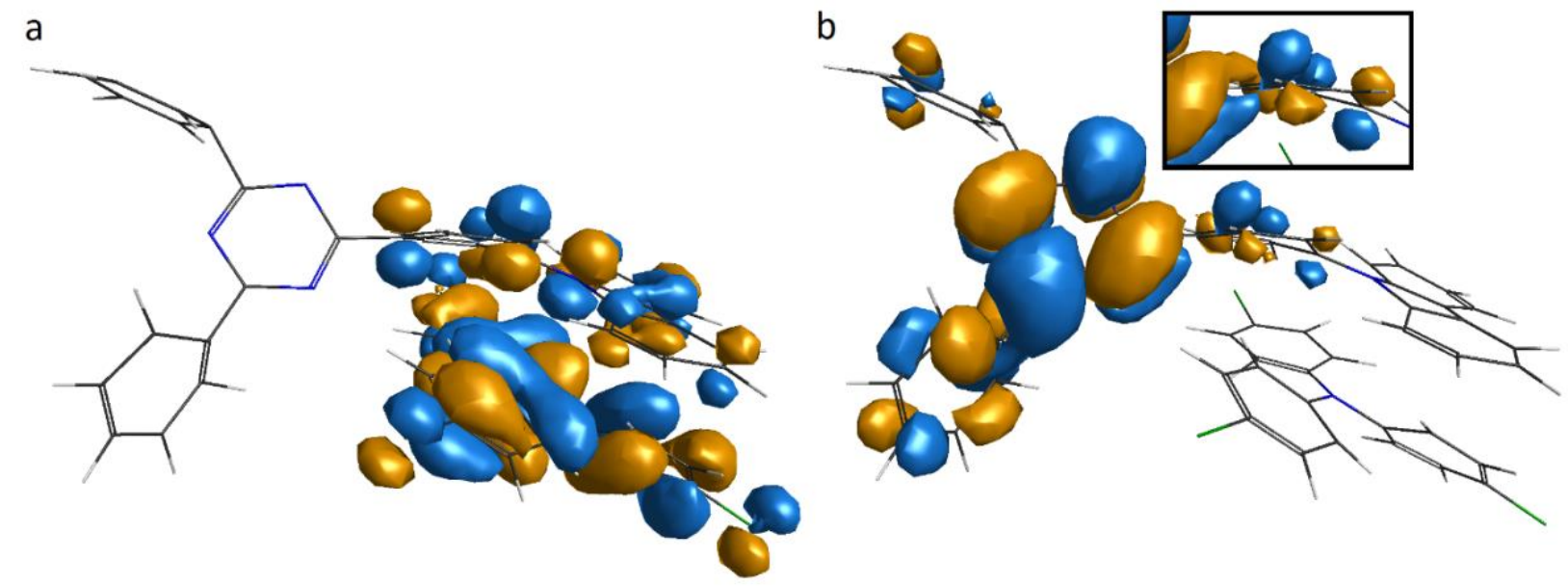

Figure $S 2$ (a) The hole and (b) the particle orbitals of the $S_{1}$ state. The insets, if any, are the corresponding NTOs of the $\mathrm{T}_{1}$ state.

Figure S3 corresponds to frame 749 in Table S6. Notice that the donor and the acceptor molecules are no longer stacked on top of each other. The hole orbital of the $S_{1}$ state, which is localized on the donor molecule, and the delocalized hole orbital of the $T_{1}$ state demonstrate that the $T_{1}$ and the $S_{1}$ states can have disparate wavefunctions. 


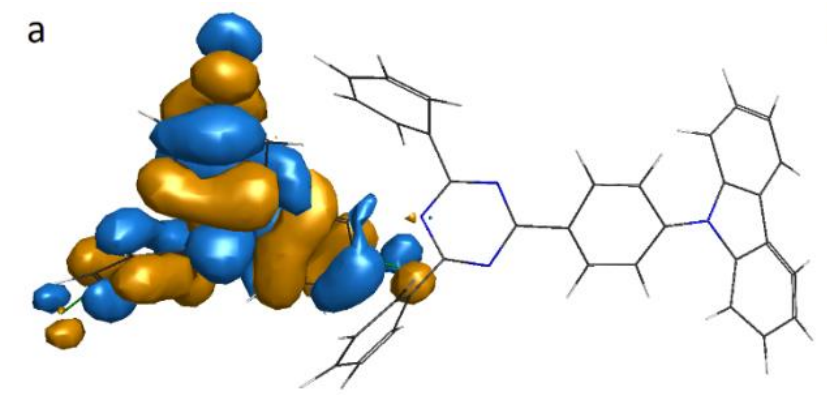

b
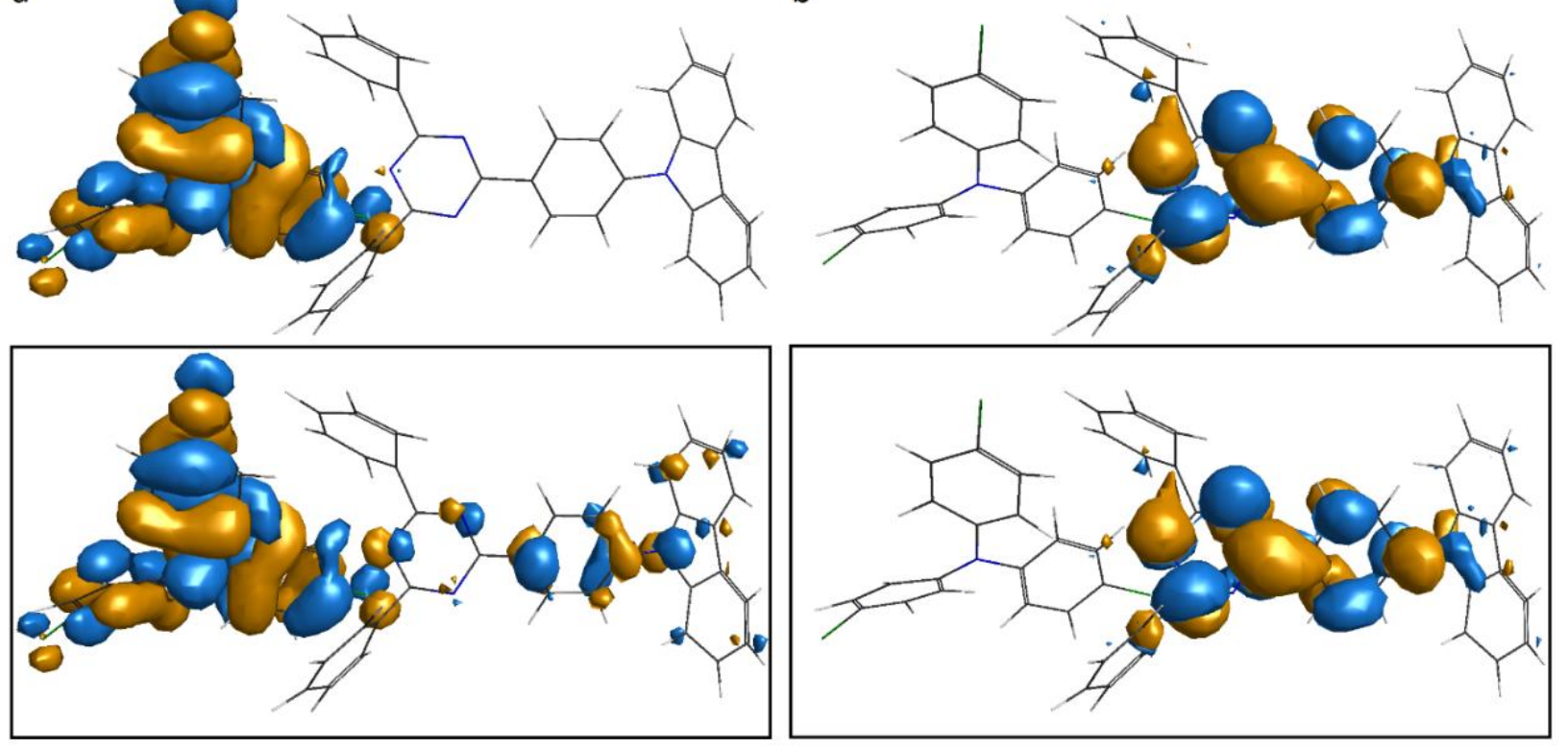

Figure S3 (a) The hole and (b) the particle orbitals of the $S_{1}$ state. The insets are the corresponding NTOs of the $T_{1}$ state.

\section{Analysis of the orbital transitions}

Among the conformers that exhibited the highest TADF rates $\left(\tau_{\text {TADF }}<10 \mu \mathrm{s}\right)$, the average HOMO-LUMO transition amplitude was 0.99 in the $S_{1}$ excitation and 0.94 in the $T_{1}$ excitation. The secondary contributions often came from the HOMO-LUMO+1 and the HOMO-1-LUMO transitions, with the HOMO-LUMO+1 transition being more important in the $\mathrm{S} 1$ excitation and vice versa in the $\mathrm{T}_{1}$ excitation. Figure $\mathrm{S} 4$ depicts the relevant KS orbitals of the same conformers as in Figure 6 in the main text. The slight delocalization of the HOMO in the first conformer should not be a surprise, since the KS orbitals have been optimized in the adiabatic representation. With that said, the HOMO and the HOMO-1 of the second conformer are localized on the donor moiety and the CZ group, respectively. In either case, the HOMO-1-LUMO transition increases the LE character of the excitation, since the bulk of the HOMO-1 resides on the carbazole group of the acceptor moiety. On the other hand, the HOMO-LUMO+1 transition contributes more CT character. With cancellation between the delocalization of the HOMO and the CT contribution of the HOMOLUMO+1 transition, the HOMO-LUMO transition amplitude should provide a reasonable relative measure of the CT/LE character. 

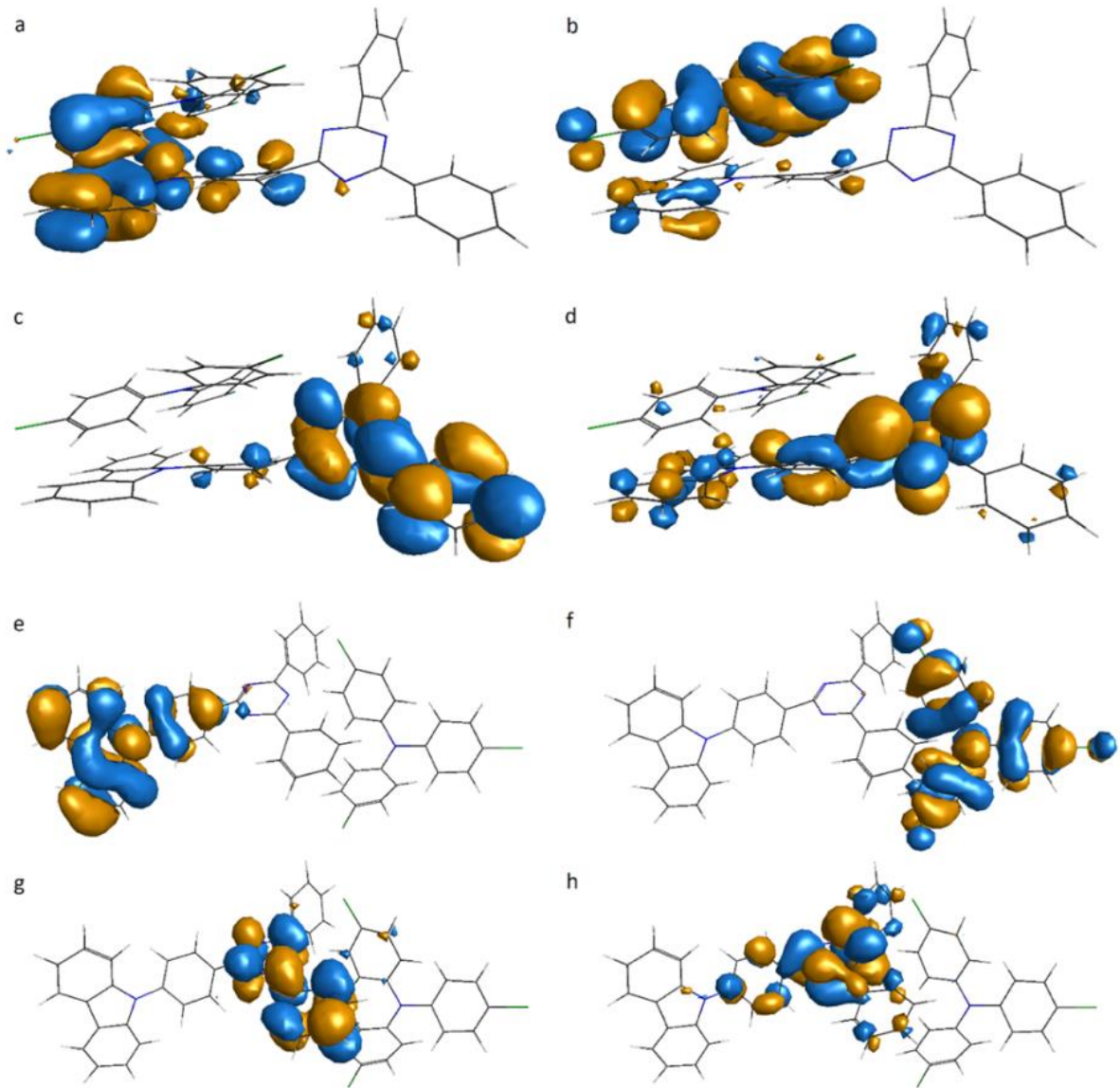

h

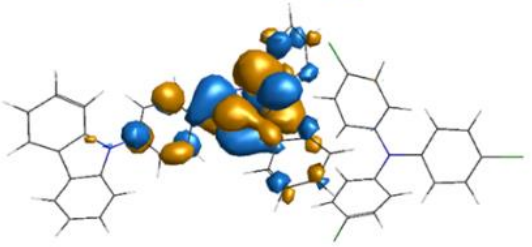

Figure S4 Kohn-Sham orbitals: (a, e) HOMO-1, (b, d) HOMO, (c, f) LUMO, and (d, h) LUMO+1.

On the other end of the spectrum, among the conformers that exhibited low TADF rates ( $\tau_{\text {TADF }}>1 \mathrm{~ms}$ ), the transitions became much more complicated, making detailed interpretation difficult. Fortunately, the KS orbitals from HOMO-1 to LUMO+1 retained their characteristics throughout the conformation space, so some insight could be extracted in terms of the HOMO-LUMO transition. In the $\mathrm{S}_{1}$ excitation, the HOMOLUMO transition still dominated, although the average transition amplitude was reduced to 0.94. Meanwhile, the $T_{1}$ excitation contained varying amounts of the HOMO-LUMO transition. In fact, some conformations exhibited negligible HOMO-LUMO transition in their $\mathrm{T}_{1}$ excitation. In such cases, there had been a switch in the energy ordering of the CT-like and the LE-like triplet states, and some higher triplet state could be found with a dominant HOMO-LUMO transition. Due to the decreased CT character in the $S_{1}$ and the $T_{1}$ states, more than $80 \%$ of these conformers had an oscillator strength $>10^{-3}$, but they also had a $\Delta \mathrm{E}_{S T}>100 \mathrm{meV}$. The change in the limiting factor of TADF from the oscillator strength to the $\Delta \mathrm{E}_{S T}$ highlights that the $S_{1}$ and the $T_{1}$ states should contain some LE character, but no more than a small fraction. 


\section{Polarizable Continuum Model}

The condensed phase effects were examined at the level of linear-response conductor-like polarizable continuum model (LR-CPCM). ${ }^{16,17}$ On the same set of conformations as the gas phase calculations, TDDFT/LR-CPCM calculations were carried out, assuming a dielectric constant of 3.0, which is quite common in the OLED matrix materials. ${ }^{18}$ The results have been summarized in Figure S5. While LR-CPCM tended to stabilize the $S_{1}$ and the $T_{1}$ states, decrease $\Delta E_{S T}$, and increase the oscillator strength, it was not enough to induce qualitative changes in the statistics.
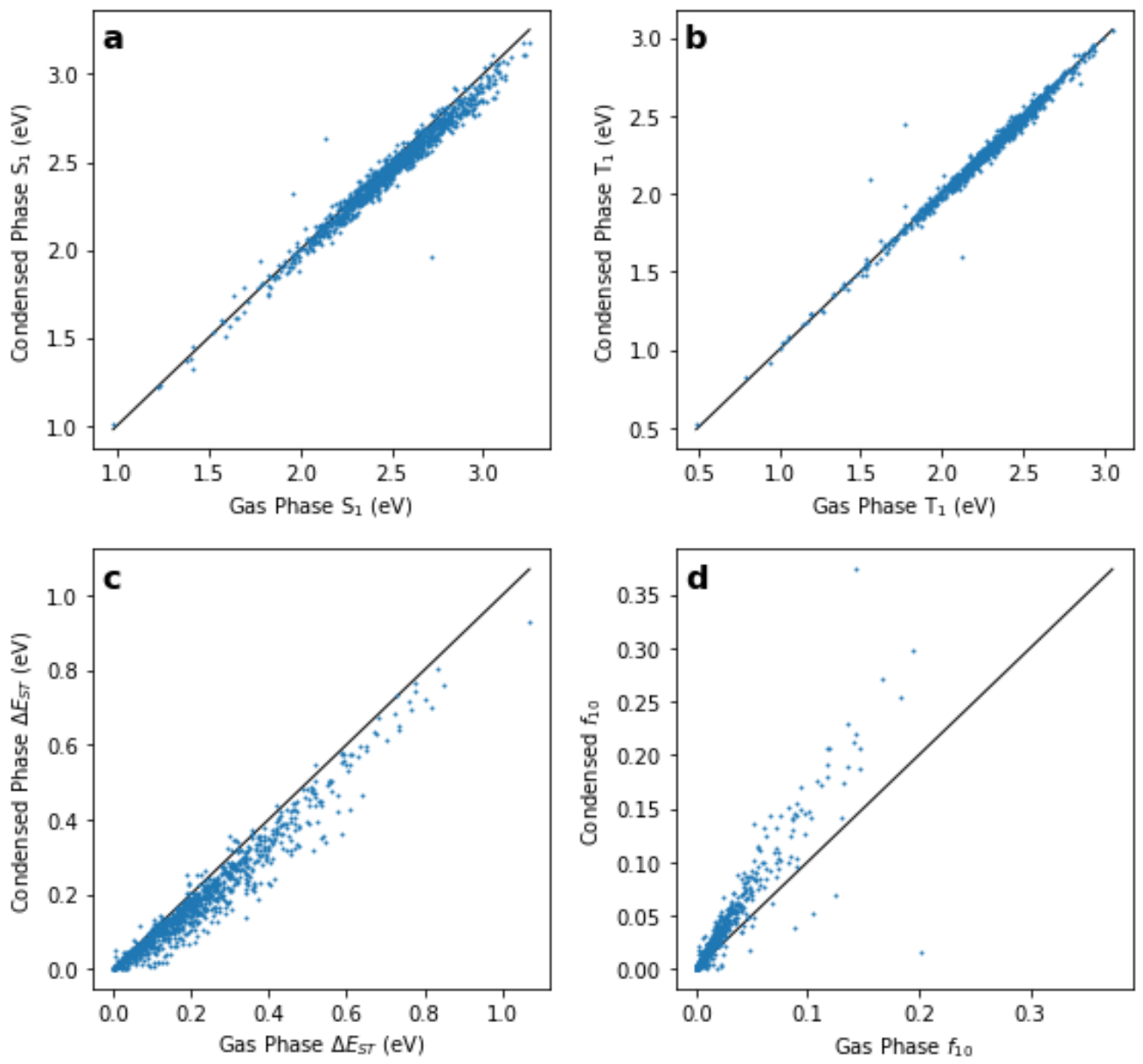

Figure S5 Scatterplots of the condensed phase against the gas phase (a) $\mathrm{S}_{1}$ excitation energy, (b) $\mathrm{T}_{1}$ excitation energy, (c) $\Delta E_{S T}$, and (d) $S_{1}-S_{0}$ oscillator strength. The black lines have unit slope $(\boldsymbol{y}=\boldsymbol{x})$. 


\section{References}

(1) Lee, K.; Kim, D. Local-Excitation versus Charge-Transfer Characters in the Triplet State: Theoretical Insight into the Singlet-Triplet Energy Differences of Carbazolyl-Phthalonitrile-Based Thermally Activated Delayed Fluorescence Materials. J. Phys. Chem. C 2016, 120 (49), 28330-28336.

(2) Becke, A. D. Density-functional Thermochemistry. III. The Role of Exact Exchange. J. Chem. Phys. 1993, 98 (7), 5648-5652.

(3) Clark, T.; Chandrasekhar, J.; Spitznagel, G. W.; Schleyer, P. v R. Efficient Diffuse Function-Augmented Basis Sets for Anion Calculations. III. The 3-21+G Basis Set for First-Row Elements, Lithium to Fluorine. J. Comput. Chem. 1983, 4 (3), 294-301.

(4) Francl, M. M.; Pietro, W. J.; Hehre, W. J.; Binkley, J. S.; Gordon, M. S.; DeFrees, D. J.; Pople, J. A. SelfConsistent Molecular Orbital Methods. XXIII. A Polarization-Type Basis Set for Second-Row Elements. J. Chem. Phys. 1982, 77 (7), 3654-3665.

(5) Gill, P. M. W.; Johnson, B. G.; Pople, J. A.; Frisch, M. J. The Performance of the Becke-Lee-Yang-Parr (B-LYP) Density Functional Theory with Various Basis Sets. Chem. Phys. Lett. 1992, 197 (4,5), 499505.

(6) Hariharan, P. C.; Pople, J. A. The Influence of Polarization Functions on Molecular Orbital Hydrogenation Energies. Theor. Chim. Acta 1973, 28 (3), 213-222.

(7) Krishnan, R.; Binkley, J. S.; Seeger, R.; Pople, J. A. Self-Consistent Molecular Orbital Methods. XX. A Basis Set for Correlated Wave Functions. J. Chem. Phys. 1980, 72 (1), 650-654.

(8) Shao, Y.; Gan, Z.; Epifanovsky, E.; Gilbert, A. T. B.; Wormit, M.; Kussmann, J.; Lange, A. W.; Behn, A.; Deng, J.; Feng, X.; et al. Advances in Molecular Quantum Chemistry Contained in the Q-Chem 4 Program Package. Mol. Phys. 2015, 113 (2), 184-215.

(9) Breneman, C.; Wiberg, K. Determining Atom-Centered Monopoles from Molecular Electrostatic Potentials. The Need for High Sampling Density in Formamide Conformational Analysis. J. Comput. Chem. 1990, 11 (3), 361-373.

(10) Jorgensen, W. L.; Maxwell, D. S.; Tirado-Rives, J. Development and Testing of the OPLS All-Atom Force Field on Conformational Energetics and Properties of Organic Liquids. J. Am. Chem. Soc. 1996, 118 (45), 11225-11236.

(11) Abraham, M. J.; Murtola, T.; Schulz, R.; Páll, S.; Smith, J. C.; Hess, B.; Lindah, E. Gromacs: High Performance Molecular Simulations through Multi-Level Parallelism from Laptops to Supercomputers. SoftwareX 2015, 1-2, 19-25.

(12) Nosé, S. A Unified Formulation of the Constant Temperature Molecular Dynamics Methods. J. Chem. Phys. 1984, 81 (1), 511-519.

(13) Nosé, S. A Molecular Dynamics Method for Simulations in the Canonical Ensemble. Mol. Phys. 2002, 100 (1), 191-198.

(14) Hoover, W. G. Canonical Dynamics: Equilibrium Phase-Space Distributions. Phys. Rev. A 1985, 31 (3), 1695-1697. 
(15) Zhang, Q.; Li, B.; Huang, S.; Nomura, H.; Tanaka, H.; Adachi, C. Efficient Blue Organic Light-Emitting Diodes Employing Thermally Activated Delayed Fluorescence. Nat. Photonics 2014, 8 (4), 326-332.

(16) You, Z. Q.; Mewes, J. M.; Dreuw, A.; Herbert, J. M. Comparison of the Marcus and Pekar Partitions in the Context of Non-Equilibrium, Polarizable-Continuum Solvation Models. J. Chem. Phys. 2015, $143(20)$.

(17) Mewes, J. M.; You, Z. Q.; Wormit, M.; Kriesche, T.; Herbert, J. M.; Dreuw, A. Experimental Benchmark Data and Systematic Evaluation of Two a Posteriori, Polarizable-Continuum Corrections for Vertical Excitation Energies in Solution. J. Phys. Chem. A 2015, 119 (21), 5446-5464.

(18) Sun, H.; Hu, Z.; Zhong, C.; Chen, X.; Sun, Z.; Brédas, J. L. Impact of Dielectric Constant on the SingletTriplet Gap in Thermally Activated Delayed Fluorescence Materials. J. Phys. Chem. Lett. 2017, 8 (11), 2393-2398. 
SI Extracting Design Principles for Efficient Thermally Activ... (1.03 MiB) view on ChemRxiv • download file 